\title{
Competition between antiferromagnetic and charge order in the Hubbard-Holstein model
}

\author{
Johannes Bauer ${ }^{1}$ and Alex C. Hewson ${ }^{2}$ \\ ${ }^{1}$ Max-Planck Institute for Solid State Research, Heisenbergstr.1, 70569 Stuttgart, Germany and \\ ${ }^{2}$ Department of Mathematics, Imperial College, London SW7 2AZ, United Kingdom
}

(Dated: November 23, 2018)

\begin{abstract}
We study the competition between an instantaneous local Coulomb repulsion and a boson mediated retarded attraction, as described by the Hubbard-Holstein model. Restricting to the case of half filling, the ground-state phase diagram and the transitions from antiferromagnetically ordered states to charge ordered states are analyzed. The calculations are based on the model in large dimensions, so that dynamical mean field theory can be applied, and the associated impurity problem is solved using the numerical renormalization group method. The transition is found to occur when electron-electron coupling strength $U$ and the induced interaction $\lambda$ due to electron-phonon coupling approximately coincide, $U \simeq \lambda$. We find a continuous transition for small coupling and large $\omega_{0}$, and a discontinuous one for large coupling and/or small $\omega_{0}$. We present results for the order parameters, the static expectation values for the electrons and phonons, and the corresponding spectral functions. They illustrate the different types of behavior to be seen near the transitions. Additionally, the quasiparticle properties are calculated in the normal state, which leads to a consistent interpretation of the low energy excitations.
\end{abstract}

PACS numbers: 71.10.Fd, 71.27.+a,71.30.+h,75.20.-g, 71.10.Ay

\section{INTRODUCTION}

A feature of strongly correlated systems is the existence of competing interactions on low energy scales which can lead to different types of symmetry breaking and different ground states. There can be various forms of magnetic order, superconducting or charge ordered states; there may also be transitions between these states, and in some cases they even coexist. For instance, compounds such as the vanadites ${ }^{\underline{1}}$, high $T_{c}$ cuprates $^{2}$, fullerides ${ }^{3}$, manganites 4.5 and organic salts $\underline{6}$ possess rather involved phase diagrams and, to understand them, an analysis of the competition between the different interactions will be important.

Here we study the competing effects between an instantaneous local Coulomb repulsion and the retarded interaction induced by a coupling to an optical phonon mode using the Hubbard-Holstein (HH) model. We consider the competition between two types of order, antiferromagnetic (AFM) and charge order (CO), which can occur in the model at half filling. The emphasis will be on treating the phonons fully quantum mechanically and in allowing for arbitrary coupling strengths, so that the full interaction parameter regime can be investigated. This is possible if we use the infinite dimensional version of Hubbard-Holstein model so that we can apply the dynamical mean field theory (DMFT), which becomes exact in this limit. The numerical renormalization group (NRG) method is then used to solve the associated effective impurity problem. This permits one to handle both strong electron-electron and strong electron-phonon interactions as well as a wide range of phonon frequencies. We focus on the ground state and spectral properties of electrons and phonons at zero temperature.

The infinite dimensional Hubbard, Holstein and com- bined $\mathrm{HH}$ models have received considerable attention in the past ${ }^{7-22}$. For the pure Holstein case, Freericks et al,$\underline{7.8}$ found instabilities to charge order and superconductivity by quantum Monte Carlo (QMC) and iterated perturbation theory for different filling factors. At half filling, Benedetti and Zeyher $\stackrel{10}{ }$, and Hague and D'Ambrumenil23 ${ }^{23}$, investigated the normal state and found a breakdown of Migdal-Eliashberg theory when a lattice instability develops for stronger electron-phonon coupling. The charge ordered ground state and phase diagram in the adiabatic limit has been analyzed by Ciuchi et al. $\underline{11}$. It was shown there that the weak and strong coupling CO states are smoothly connected.

For the Hubbard-Holstein model in the absence of long range order, the phase diagram of the paramagnetic $(\mathrm{PM})$, bipolaronic (BP) phases and the metal-insulator (MI) transition has been established ${ }^{16,24}$. Another recent study of the model without long range order deals with the topic of polaron formation 18,19 with finite electron density, extending the original work of Holstein ${ }^{25}$ who considered the single electron case only. The occurrence of superconductivity was studied in Ref. 9, 20.21 . In a two site calculation Takada found superconductivity with off-site pairing at half filling in a very small parameter regime in the antiadiabatic region 21 . The effect of phonons on the quasiparticle excitations in the presence of AFM has also been investigated 26 . There have also been extensive calculations for the one dimensional version of model27? -31 , which we shall comment on briefly later.

Our analysis here of the HH model will extend the earlier work by allowing for AFM and CO states, which are the dominant instabilities at half filling. We study the transitions between these states. This will give a more complete picture of the phase diagram and the proper- 
ties of the model in the ordered phases. In the regions of the phase diagram with $\mathrm{CO}$, we also obtained superconducting solutions, but the CO states were found to have lower energy. We calculate the static and dynamic properties in both these types of broken symmetry phases. The paper is structured as follows. In Sec. II we specify the formal setup of the HH model and the DMFT-NRG method. We also give explicit expressions for the different contributions to the total energy. The dependence of these on the interactions is discussed in detail in Sec. IV. Before that in Sec. III, we discuss the global phase diagram, the order parameters and static quantities and their dependence on $U, \lambda$ and $\omega_{0}$. Sec. $\mathrm{V}$ explores the normal state properties of the HH model which helps to understand the ground state phase diagram and transition. In Sec. VI we discuss how the bosonic properties are modified by the coupling to the electronic system. In Sec. VII we present results for the electronic and bosonic spectral functions, before concluding in Sec. VIII.

\section{MODEL AND DMFT-NRG SETUP}

The Hamiltonian for the $\mathrm{HH}$ model is given by

$$
\begin{aligned}
H= & -\sum_{i, j, \sigma}\left(t_{i j} c_{i, \sigma}^{\dagger} c_{j, \sigma}+\text { h.c. }\right)+U \sum_{i} \hat{n}_{i, \uparrow} \hat{n}_{i, \downarrow} \\
& +\omega_{0} \sum_{i} b_{i}^{\dagger} b_{i}+g \sum_{i}\left(b_{i}+b_{i}^{\dagger}\right)\left(\sum_{\sigma} \hat{n}_{i, \sigma}-1\right) .
\end{aligned}
$$

$c_{i, \sigma}^{\dagger}$ creates an electron at lattice site $i$ with spin $\sigma$, and $b_{i}^{\dagger}$ a phonon with oscillator frequency $\omega_{0}, \hat{n}_{i, \sigma}=c_{i, \sigma}^{\dagger} c_{i, \sigma}$. The electrons interact locally with strength $U$, and their density is coupled to an optical phonon mode with coupling constant $g$. We have set the ionic mass to $M=1$ in (11). The local oscillator displacement is related to the bosonic operators by $\hat{x}_{i}=\left(b_{i}+b_{i}^{\dagger}\right) / \sqrt{2 \omega_{0}}$, where $\hbar=1$, and one can define a characteristic length $x_{0}=1 / \sqrt{\omega_{0}}$ for the oscillator. In Appendix $\mathrm{A}$ we give the details for a mean field calculation in the adiabatic limit for this model.

For our calculations we assume a bipartite lattice with $A$ and $B$ sublattice, where the matrix Green's function can be written in the form

$$
\underline{G}_{\boldsymbol{k}, \sigma}(\omega)=\frac{1}{\zeta_{A, \sigma}(\omega) \zeta_{B, \sigma}(\omega)-\varepsilon_{\boldsymbol{k}}^{2}}\left(\begin{array}{cc}
\zeta_{B, \sigma}(\omega) & \varepsilon_{\boldsymbol{k}} \\
\varepsilon_{\boldsymbol{k}} & \zeta_{A, \sigma}(\omega)
\end{array}\right),
$$

with $\zeta_{\alpha, \sigma}(\omega)=\omega+\mu_{\alpha, \sigma}-\Sigma_{\alpha, \sigma}(\omega), \alpha=A, B$, and $\boldsymbol{k}$-independent self-energy ${ }^{32}$. For commensurate charge order we have $\mu_{A, \sigma}=\mu-h_{c}, \mu_{B, \sigma}=\mu+h_{c}$ and $\Sigma_{B, \sigma}(\omega)=U n-\Sigma_{A, \sigma}(-\omega)^{*}$, with $n=\left(n_{A}+n_{B}\right) / 2$, $n_{\alpha}=\sum_{\sigma} n_{\alpha, \sigma}, n_{\alpha, \sigma}=\left\langle\hat{n}_{\alpha, \sigma}\right\rangle$. For the AFM order one has $\mu_{A, \sigma}=\mu-\sigma h_{s}, \mu_{B, \sigma}=\mu+\sigma h_{s}$, and the condition $\Sigma_{B, \sigma}(\omega)=\Sigma_{A,-\sigma}(\omega)$. We consider solutions of exclusive AFM or CO, where the symmetry breaking fields vanish, $h_{c}, h_{s} \rightarrow 0$.
In the case with symmetry breaking, the effective Weiss field is a $2 \times 2$ matrix $\underline{\mathcal{G}}_{0}^{-1}(t)$. The DMFT self-consistency equation in this case reads ${ }^{33}$

$$
\underline{\mathcal{G}}_{0, \sigma}^{-1}(\omega)=\underline{G}_{\sigma}(\omega)^{-1}+\underline{\Sigma}_{\sigma}(\omega)
$$

The matrix of local lattice Green's functions $\underline{G}_{\sigma}(\omega)=$ $1 / N \sum_{k} \underline{G}_{k, \sigma}(\omega)$ is obtained by integrating over the density of states, $1 / N \sum_{\boldsymbol{k}} f\left(\varepsilon_{\boldsymbol{k}}\right)=\int \mathrm{d} \varepsilon \rho_{0}(\varepsilon) f(\varepsilon)$. We as-

sume a semi-elliptic DOS, $\rho_{0}(\varepsilon)=2 \sqrt{D^{2}-\varepsilon^{2}} / \pi D^{2}$ corresponding to a Bethe lattice in all the following calculations. In the DMFT this local Green's function, and the self-energy are identified with the corresponding quantities for an effective impurity mode $\mathrm{l}^{33}$. One focuses for the calculations on the properties of the $A$-sublattice. We can take the form of this impurity model to correspond to an Anderson-Holstein impurity mode ${ }^{34}$ and calculations are carried out as detailed, for instance in Ref. 35, 36. We solve the effective impurity problem with the numerical renormalization group 37.38 (NRG) adapted to these cases with symmetry breaking. The NRG has been shown to be very successful for calculating the local dynamic response functions, and we use the recent approach ${ }^{39.40}$ based on the complete basis set proposed by Anders and Schiller $\underline{41}$. For the logarithmic discretization parameter we take the value $\Lambda=1.8$ and keep about 1000 states at each iteration. The bosonic Hilbert space is restricted to a maximum of 50 states.

In the AFM case the $A$-sublattice magnetization, $\Phi_{\text {afm }}=m_{A}=\left(n_{A, \uparrow}-n_{A, \downarrow}\right) / 2$ serves as an order parameter. For $\mathrm{CO}$ we define $\Phi_{\mathrm{co}}=\left(n_{A}-1\right) / 2$.

To find the ground state of the system we calculate the ground state energy $E_{\text {tot }}=\langle H\rangle / N$ of the HH Hamiltonian (11) in the different phases. This gives generally,

$$
E_{\mathrm{tot}}=E_{\mathrm{kin}}+E_{U}+E_{g}+E_{\mathrm{ph}} .
$$

The first term is the kinetic energy, which reads

$$
E_{\mathrm{kin}}=\sum_{\sigma} \int \mathrm{d} \varepsilon_{\boldsymbol{k}} \rho_{0}\left(\varepsilon_{\boldsymbol{k}}\right) \varepsilon_{\boldsymbol{k}} \int \mathrm{d} \omega f(\omega) \rho_{A B, \boldsymbol{k}, \sigma}(\omega),
$$

where $\rho_{A B, \boldsymbol{k}, \sigma}(\omega)=-\operatorname{Im} G_{A B, \boldsymbol{k}, \sigma}(\omega) / \pi$ for the offdiagonal Green's function in (2) and $f(\omega)$ is the Fermi function, where $f(\omega)=\theta(-\omega)$ at zero temperature. In the non-interacting case this expression can be evaluated analytically and we find for half filling, $\mu=0$, $E_{\text {kin }}^{0}=-4 D / 3 \pi$, which for $D=2$ is $E_{\text {kin }}^{0} \simeq-0.849$. This can be used as reference energy. More specifically one finds

$$
E_{\text {kin }}=\sum_{\sigma} \int \mathrm{d} \varepsilon_{\boldsymbol{k}} \rho_{0}\left(\varepsilon_{\boldsymbol{k}}\right) \varepsilon_{\boldsymbol{k}} \int \mathrm{d} \omega f(\omega) g_{\boldsymbol{k}, \sigma}(\omega)
$$

where

$$
g_{\boldsymbol{k}, \sigma}(\omega)=-\frac{1}{\pi} \operatorname{Im} \frac{1}{\sqrt{\zeta_{A, \sigma}(\omega) \zeta_{B, \sigma}(\omega)}-\varepsilon_{\boldsymbol{k}}} .
$$


The interaction energies $E_{U}, E_{g}$ can be calculated from expectation values,

$$
E_{U}=\frac{U}{2} \sum_{\alpha}\left\langle\hat{n}_{\alpha, \uparrow} \hat{n}_{\alpha, \downarrow}\right\rangle, E_{g}=\frac{g}{2} \sum_{\alpha}\left\langle\left(b_{\alpha}+b_{\alpha}^{\dagger}\right)\left(\hat{n}_{\alpha}-1\right)\right\rangle
$$

We distinguish between $A$ - and $B$-sublattice values, which are equal in the AFM case, but not for the CO case. There we use the operator identity $\hat{n}_{B, \sigma}=1-\hat{n}_{A, \sigma}$ (particle hole transformation), at half filling, which yields $\left\langle\hat{n}_{B, \uparrow} \hat{n}_{B, \downarrow}\right\rangle=\left\langle\hat{n}_{A, \uparrow} \hat{n}_{A, \downarrow}\right\rangle+1-\left\langle\hat{n}_{A}\right\rangle$. The terms for $E_{g}$ turn out to be equal on $A$ and $B$ sublattice as both terms will contribute with opposite value.

\section{PHASE DIAGRAM AND STATIC PROPERTIES OF THE ELECTRONS}

As the electron-phonon coupling in (1) is linear, the bosonic field can be integrated out in a path integral framework, which yields a purely electronic theory with an effective electron-electron interaction of the form

$$
U_{\mathrm{eff}}(\omega)=U+g^{2} D^{0}(\omega)
$$

where the free phonon propagator on the real axis is $D^{0}(\omega)=2 \omega_{0} /\left(\omega^{2}-\omega_{0}^{2}\right)$. These are the competing interactions on different energy scales. There is a limiting case $\omega_{0} \rightarrow \infty$, the antiadiabatic limit, where $\lambda=2 g^{2} / \omega_{0}$ is kept fixed. In this limit, $U_{\text {eff }}(\omega)$ becomes independent of $\omega$ and tends to $U_{\text {eff }}=U-\lambda$, so that the model then becomes equivalent to a Hubbard model with $U=U_{\text {eff }}$. Generally, the situation is more complicated. For large $\omega$ the Coulomb repulsion $U$ is dominant in (8) as $D^{0}(\omega)$ goes to zero. However, $\omega_{0}$ enters as a relevant energy scale at lower energy and for $|\omega| \lesssim \omega_{0}$ the competition between the bare interactions is most important. The DMFT calculations deal with these competing interactions on different energy scales, and as a result the ground state phase diagram of the infinite dimensional HH model at half filling emerges. This phase diagram was already presented earlier ${ }^{42}$. In order to give a comprehensive account in this paper, we include some of these results in the following.

We comment briefly on the notation and energy scales. In electron-phonon physics, specifically in MigdalEliashberg theory, the electron phonon coupling is usually specified by a dimensionless parameter, often called $\lambda$, involving the coupling strength and an electronic scale. In contrast, in this paper $\lambda$ has the dimension of an energy, and it is compared with the Coulomb repulsion $U$. The dimensionless parameter for electron-phonon coupling corresponding to the usual convention would be $\bar{\lambda}=\rho_{0}(0) \lambda$, where $\rho_{0}(0)=2 /(D \pi)$. For physical optical phonons the scale $\omega_{0}$ is expected to be roughly $\omega_{0} \sim 0.01 W-0.2 W$, where $W$ is the electronic bandwidth. The purpose of the paper is to characterize the electron-phonon system quite generally, with a tunable parameter $\omega_{0}$, which for many results we chose as $\omega_{0}=0.15 \mathrm{~W}$ neither too close to the adiabatic nor to the antiadiabatic limiting cases for illustration of intermediate behavior. It should be noted that the value is large for most realistic electron-phonon systems with the possible exception of the alkali doped fullerides ${ }^{43}$. For the former cases smaller values such as $\omega_{0}=0.05 \mathrm{~W}$ could serve as a better guideline.

\section{A. Phase diagram}

We first present the complete phase diagram as shown in Fig. 1. The overall energy scale is set by the bandwidth $W=4 t=4$, and the phonon frequency $\omega_{0}=0.6 t$ was chosen. Many results such as the global phase diagram are similar for different choices of $\omega_{0}$, but we will also point out the differences appearing for other values of $\omega_{0}$. The limiting cases of the phase diagram can be understood on a qualitative level. The $U$-axis corresponds to the pure repulsive Hubbard model which is known to be AFM ordered at weak coupling $\underline{44}$ for a bipartite lattice, and this order is smoothly connected to the strong coupling Heisenberg AFM ${ }^{35,36}$. Also along the $\lambda$-axis, the corresponding pure Holstein model has a charge ordered ground state for $g>0$ and $\omega_{0}>0$, such that the limits of weak and strong coupling are smoothly connected ${ }^{7}$. For finite $U$ and $g$ we find that the transition line is close to the line $\lambda=U$ with a small tendency towards $\lambda>U$. For larger values of the interactions we have included a dashed line $(\diamond)$ above which our DMFT-NRG calculations find solutions with finite $\Phi_{\text {co }}$ and another one (o) below which $\Phi_{\text {afm }}$ is finite.

For weaker coupling $(U<3)$ the order parameters become very small close to the line $U_{\text {eff }}=0$. There are strong indications that the transition proceeds both directly and continuously from one type of ordered to the other ordered state, such that only at the critical point both order parameters vanish. For instance, we find that all the relevant response quantities behave continuously. A direct order to order transition is found in the antiadiabatic limit, where for any finite $U_{\text {eff }}>0$ the system is AFM ordered ${ }^{44}$ and for $U_{\text {eff }}<0$ in the CO or SC state ${ }^{45}$. We have therefore a continuous transition from an ordered to an ordered state with vanishing order parameters at the transition. There is no reason to expect that the transition becomes discontinuous immediately when $\omega_{0}$ is decreased from infinity to a finite value $\omega_{0} \gg W$. In addition, in the DMFT-NRG calculations we find that near the transitio, the smaller $\omega_{0}$ is, the larger the order parametern becomes, as detailed later in Fig. 7. Thus we conclude that the direct continuous transition scenario persists for weak coupling and finite $\omega_{0}$. Mean field calculations (see Appendix $\mathrm{A}$ ) in the adiabatic limit support the picture of a direct transition from one ordered state to the other, however, the transition is always discontinuous then. Also the local effective quasiparticle interaction $U^{r}$ (which will be discussed more fully later) is observed to change sign at $U_{\text {eff }}=0$, which is consistent 


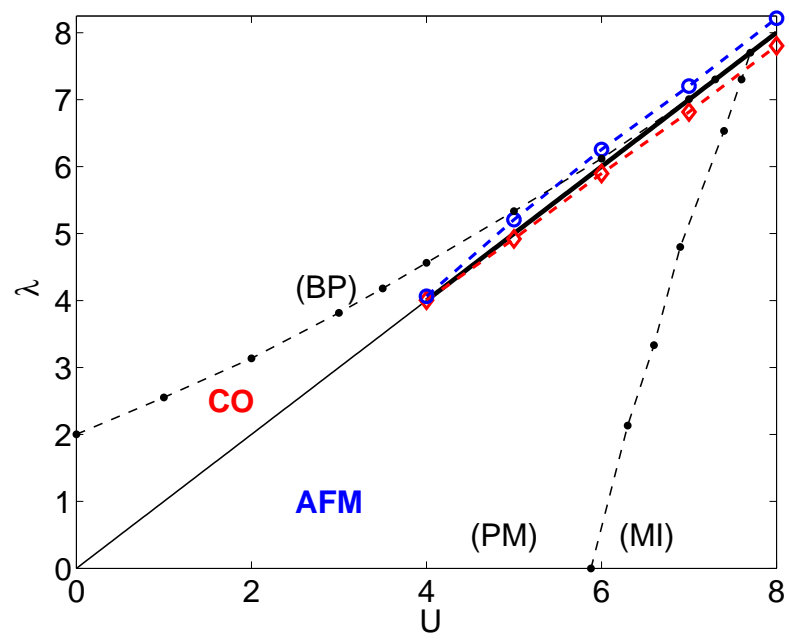

FIG. 1: (Color online) The phase diagram of antiferromagnetic (AFM) and charge order (CO) in the $U$ - $\lambda$-plane. The thin black line $\lambda \simeq U$ gives a continuous transition and the thick line a discontinuous one. A non-zero order parameter was found above dashed line with diamonds for $\mathrm{CO}$ and below the dashed line with circles for AFM order. The dashed lines with points gives the transition for phases with no long range order, a paramagnetic metallic (PM), bipolaronic (BP) and Mott insulator (MI).

with a change of ground state there.

For larger couplings we have a parameter regime where we can find finite $\Phi_{\text {afm }}$ and $\Phi_{\text {co }}$. In this regime the transition from the AFM to the CO turns out to be discontinuous and one can identify a point on the transition where the nature of transition changes. The calculation of the total ground state energy (see inset of Fig. 4) shows that also here the transition occurs approximately at $U_{\text {eff }} \simeq 0$. The behavior of the model along the line $U=\lambda$ has been studied in Ref. ${ }^{46}$.

The HH model in one dimension has been studied in great detail with efficient numerical methods ${ }^{27-29}$. One finds a Mott insulator with strong antiferromagnetic correlations, but no long range order, when $U_{\text {eff }}>0$, and a Peierls charge density wave (CDW) insulator for $U_{\text {eff }}<0$. There is, however, a metallic region with finite spin gap, but no charge gap in between these two phases. The transition line to the CDW state appears for values of $\lambda$ a bit larger than $U$, similar to what is observed in our calculations but more pronounced. For larger $U$ this intermediate region shrinks until we get a direct first order Mott-Peierls transition. A major difference with the high dimensional results is the real symmetry breaking in our case as well as the existence of the intermediate region, for which we find no indication here. Nevertheless the mentioned similarities and also recent results in $d=2$ (adiabatic limit) ${ }^{47}$ suggest that the general features of the phase diagram in Fig. 1 might be quite general, largely independent of dimensionality.

In Fig. 1 we have also included the phase boundaries of the $\mathrm{HH}$ model when no long range order is allowed for.
We see that only for large coupling do the phase boundaries merge, while for smaller couplings other scales are important. For the case $\omega_{0}=0.2$ this has been analyzed earlier $16,17,24$. The Mott transition, as obtained on increasing $U$ for fixed $g$ is only little affected by the additional electron-phonon coupling, which is manifested in a shift of the critical $U_{c}$ for the transition. The metalbipolaron transition, observed when increasing $g$ for fixed $U$, is of second order for smaller interactions and becomes of first order for large interactions, and thus similarities with the ground state behavior are found. Note that the $\mathrm{CO}$ state and the bipolaronic state are different, as in the latter no symmetry is broken and the occupation expectation value is always 1 , whereas in the $\mathrm{CO}$ state $n_{A} \neq n_{B}$. For details we refer to Refs. 16, 17,24.

\section{B. Order parameter as a function of $\lambda$}

We consider here the way the two types of order parameter change as a function of $\lambda$ for fixed values of $U$ both in the weak coupling and strong coupling regime. A weaker coupling case with $U=2$ is shown in Fig. 2.

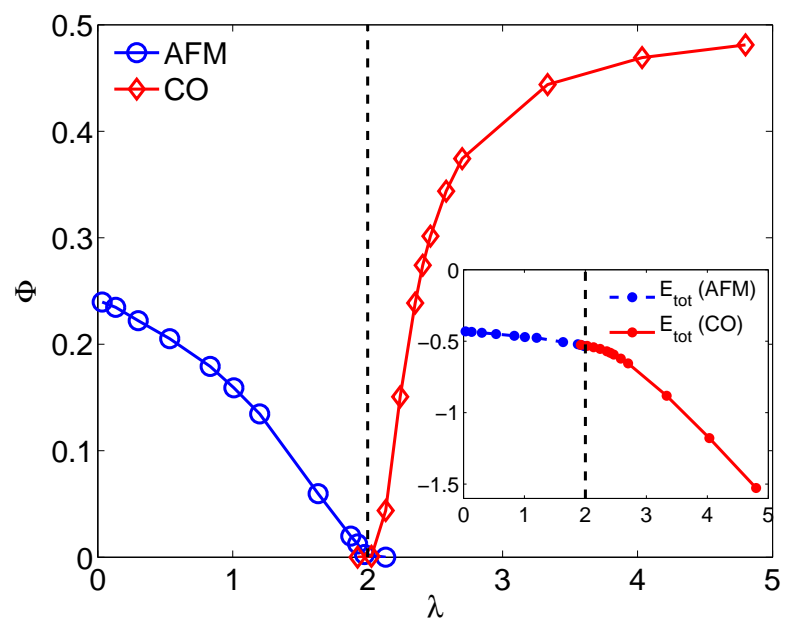

FIG. 2: (Color online) The expectation values $\Phi$ for $U=2$ as a function of $\lambda$. The inset shows the total energy.

We can see that the AFM order is decreased when the electron phonon-coupling is increased, as the repulsion is reduced. Near $\lambda=U$ the ordering scale is very small $\left(<10^{-3}\right)$ and cannot be resolved in our DMFT-NRG calculations. For $\lambda>U$ the $\Phi_{\text {co }}$ shows a steep rise with $\lambda$. For this weak coupling case we can study the limit $\omega_{0} \rightarrow 0$ and compare with the corresponding static mean field theory (details see appendix $\mathrm{A}$ ). For $U=2$ the numerical results are shown in Fig. 3.

The solutions are for situations where the order is exclusive, i.e. only one of the order parameters is nonzero. In the mean field calculation the AFM order parameter is larger than in the DMFT case, and when $\lambda$ is increased is seen not to be affected by the electron phonon cou- 


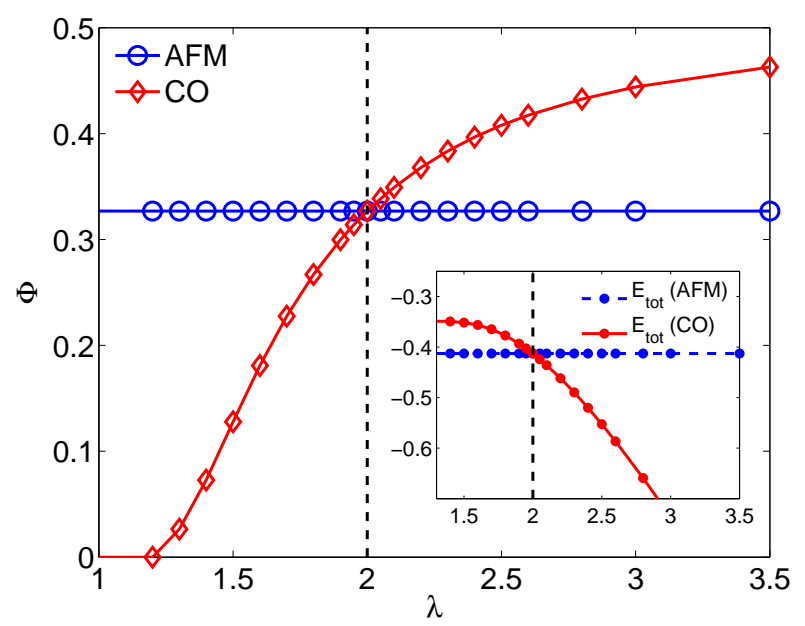

FIG. 3: (Color online) Mean field result for the order parameter and total energy shown in the inset.

pling as long as $\Phi_{\mathrm{co}}=0$. In contrast the CO order state $\Phi_{\text {co }}$ feels the $U$-term, but increases with $\lambda$. For $U=\lambda$ the mean field equations give order parameters that coincide, and once $\Phi_{\text {co }}$ exceeds $\Phi_{\text {afm }}$ the charge ordered state possesses the lowest energy as can be seen from equation (A13) and the inset in Fig. 3. We can infer from this weak coupling result that there is a direct, discontinuous transition from an ordered to an ordered state at $U=\lambda$ for $\omega_{0} \rightarrow 0$. As seen in Fig. 2 the behavior is strongly modified when quantum fluctuations are included and $\omega_{0}$ is well finite, as the order parameters are influenced much more by the presence of the competing interaction. It is numerically not possible to study the limit $\omega_{0} \rightarrow 0$ within our DMFT approach due to the increase in the bosonic Hilbert space. The analysis for different values of $\omega_{0}$ is however conform with the trends discussed here (see Fig. 7).

For larger couplings we saw that the two dashed lines in Fig. 1 cross, which means that we have a parameter regime where we find finite $\Phi_{\text {afm }}$ and $\Phi_{\text {co }}$. An example for this behavior is shown in Fig. 4 for $U=5$.

The transition here is seen to be rather sharp. The calculation of the total ground state energy (see inset) shows that the transition occurs approximately at $U_{\text {eff }}=0$, in fact it occurs for small negative $U_{\text {eff }}$, i.e., on the $\lambda>U$ side. A number of quantities such as the double occupancy $\left\langle\hat{n}_{\uparrow} \hat{n}_{\downarrow}\right\rangle$ (see section IIC) show discontinuities at the transition. The total energy (see inset of Fig. (4) is continuous function of $\lambda$. It shows, however, a kink at the transition, such that a first derivatives will be discontinuous.

\section{Double occupancy}

A characteristic quantity for the electronic part of the system is the expectation value for the local double occupancy. This is a homogeneous quantity in the normal $(\mathrm{N})$

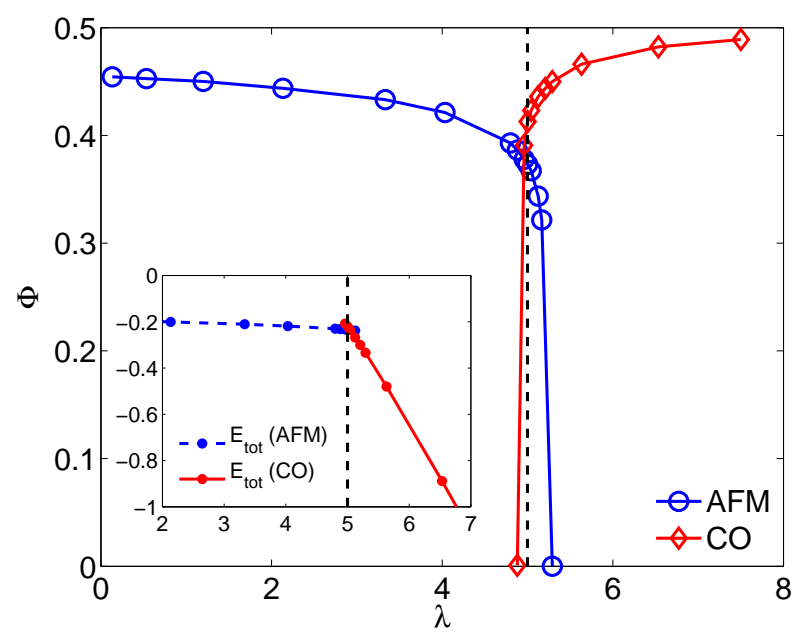

FIG. 4: (Color online) The expectation values $\Phi$ for $U=5$ as a function of $\lambda$.

and AFM phase. In the charge ordered phase it differs for $A$ - and $B$-sublattice, and $\left\langle\hat{n}_{A, \uparrow} \hat{n}_{A, \downarrow}\right\rangle$ and $\left\langle\hat{n}_{B, \uparrow} \hat{n}_{B, \downarrow}\right\rangle$ are given as detailed above when discussing the energy due to the Hubbard interaction term. We can compare the quantities by taking the average over the two sublattices, $\left\langle\hat{n}_{\uparrow} \hat{n}_{\downarrow}\right\rangle=\sum_{\alpha}\left\langle\hat{n}_{\alpha, \uparrow} \hat{n}_{\alpha, \downarrow}\right\rangle / 2$. In Fig. 5 we show the results for $U=2,5$ as a function of $\lambda$. We have included the $\mathrm{N}$, $\mathrm{AFM}$ and $\mathrm{CO}$ state, and for the latter, the averaged as well as the sublattice quantities.

For the continuous transition at $U=2$ we find a small nearly linear increase with $\lambda$ for the $\mathrm{N}$ and AFM state. The values for $\mathrm{N}$ and $\mathrm{AFM}$ state are very similar with the double occupancy being larger in the normal phase, where the charge carriers are more mobile than in the ordered phase. This is different from the strong coupling case, where the $\mathrm{N}$ state is in the Mott phase, and the double occupancy is lower than in the AFM case. This is due to the super-exchange mechanism which leads to a kinetic energy gain and a slightly higher mobility and also double occupancy. When $\lambda>U$ and charge order sets in, the double occupancy shows a steep rise on the $A$-sublattice and decrease on the $B$-sublattice. As can be seen in the top panel of Fig. [5 the $\lambda$-dependence is continuous for $U=2$. At approximately $\lambda=3$ the $\mathrm{N}$ state metal-bipolaron transition occurs, where $\left\langle\hat{n}_{\uparrow} \hat{n}_{\downarrow}\right\rangle$ in the normal state increases rapidly (but also continuously). $\left\langle\hat{n}_{\uparrow} \hat{n}_{\downarrow}\right\rangle$ is then larger than in the CO state which can be understood by thinking about the fact that the $\mathrm{CO}$ state wins energetically against the $\mathrm{BP}$ state through kinetic energy due to pair hopping (see Sec. IV).

The overall behavior in the lower panel of Fig. 5 for $U=5$ is similar. $\left\langle\hat{n}_{\uparrow} \hat{n}_{\downarrow}\right\rangle$ increases slightly with $\lambda$ for a certain range and more rapidly near the transition. There, we can clearly see the discontinuous behavior for $\lambda \simeq 5$ and $\left\langle\hat{n}_{\uparrow} \hat{n}_{\downarrow}\right\rangle$ jumps from the value 0.09 in the AFM state to 0.42 in the CO state. The BP state again has a larger double occupancy than the average of the $\mathrm{CO}$ state. 

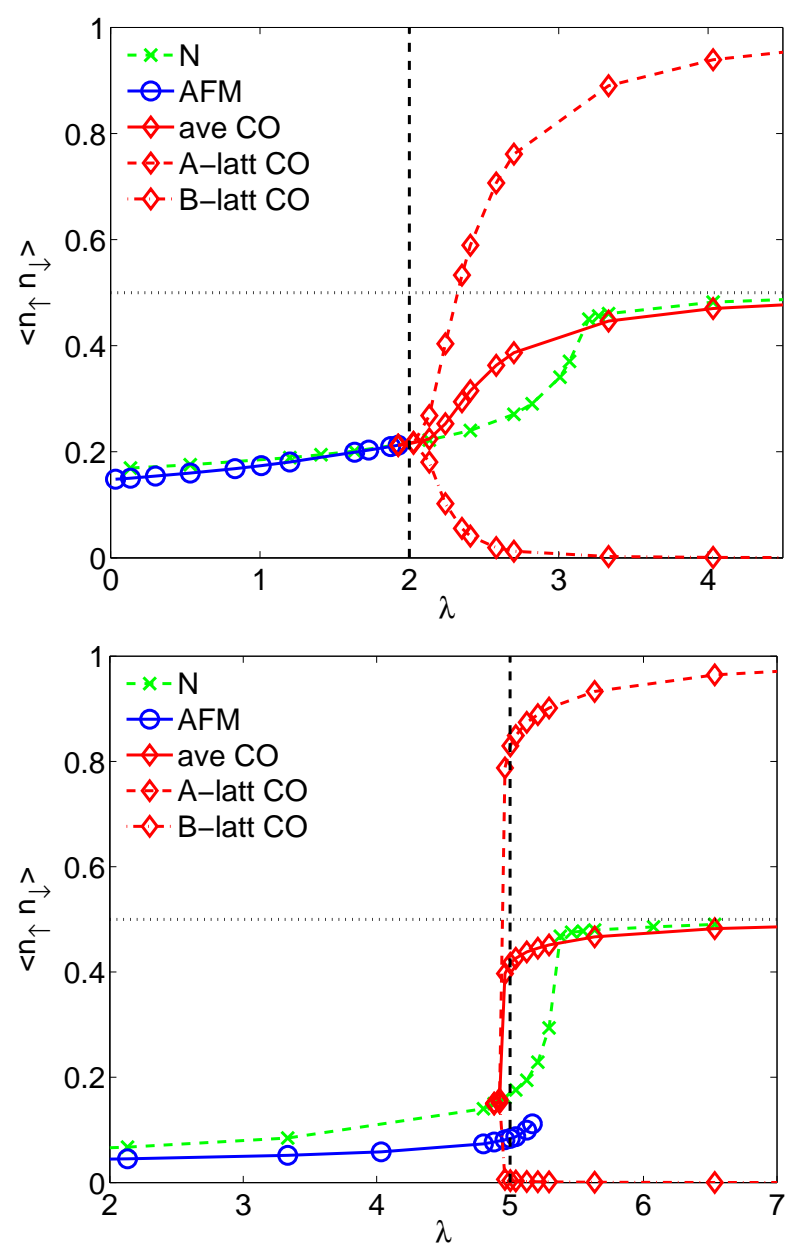

FIG. 5: (Color online) The expectation values $\left\langle n_{\uparrow} n_{\downarrow}\right\rangle$ for $U=2$ (upper panel) and $U=5$ (lower panel) as a function of $\lambda$ for the N, AFM and CO state.

\section{Order parameter as a function of $U$}

So far we have analyzed the transition as a function of $\lambda$. Similarly, we can look at the properties of the model as a function of the on-site repulsion $U$. We focus on the order parameters and look at the cases of a fixed $\lambda \simeq 2.13$ $(g=0.8)$ and $\lambda \simeq 4.8(g=1.2)$ shown in Fig. 6 ,

In both cases for small $U$ the $\mathrm{CO}$ state dominates, but on increasing $U, \Phi_{\text {co }}$ is driven to zero and $\Phi_{\text {afm }}$ becomes finite. Similar to the weaker coupling case for fixed $U$ for $\lambda \simeq 2.13$, the order parameters approach zero at the transition. We also have continuous transition here, which is visible in the total energy plotted as an inset.

In contrast for larger $\lambda \simeq 4.8$ (lower panel in Fig. 6) the picture is as for the larger coupling cases above, where the transition occurs with finite order parameters, which change discontinuously. Also here, as seen in the inset, the total ground state energy displays a kink at the transition. Summarizing, we can say that the transition occurs in similar fashion as a function of $U$ or $\lambda$. Depending on the magnitude of the coupling constants continuous or
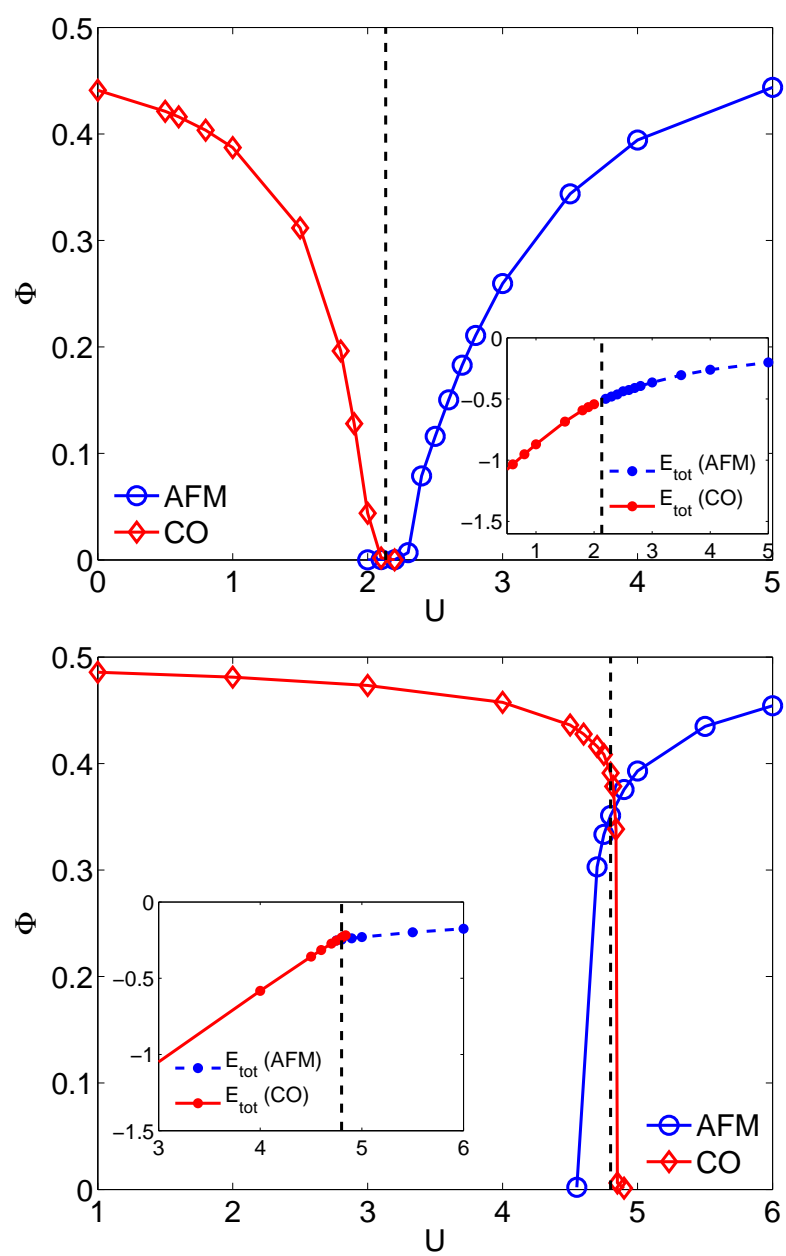

FIG. 6: (Color online) The expectation values $\Phi$ for $\lambda \simeq 2.13$ (top) and $\Phi$ for $\lambda \simeq 4.8$ (bottom) as a function of $U$. The insets show the total ground state energy.

discontinuous transitions can be observed.

\section{E. Dependence of the order parameter on $\omega_{0}$}

So far we have found that the transition from the $\mathrm{CO}$ to the AFM state occurs close to $U_{\text {eff }}=0$ independently of the other parameters. For fixed $\omega_{0}$, when studying the order parameters or double occupancy, one could see quite a different dependence on $\lambda$ or $U$ near the transition with a continuous and discontinuous behavior. In the following we study how the order parameters depend on $U_{\text {eff }}$ near the transition for different values of $\omega_{0}$. We illustrate this in Fig. 7, where we plot the respective order parameters for different $\omega_{0}$, including the case $\omega_{0} \rightarrow \infty$, which is given by the pure Hubbard model with local interaction $U_{\text {eff }}$. We have held $U=3$ fixed and varied $g$ to obtain the desired values.

As one can see clearly the "sharpness" of the transition increases when $\omega_{0}$ is decreased. For $\omega_{0} \rightarrow \infty$ the order parameters approach zero in a similar exponential form 


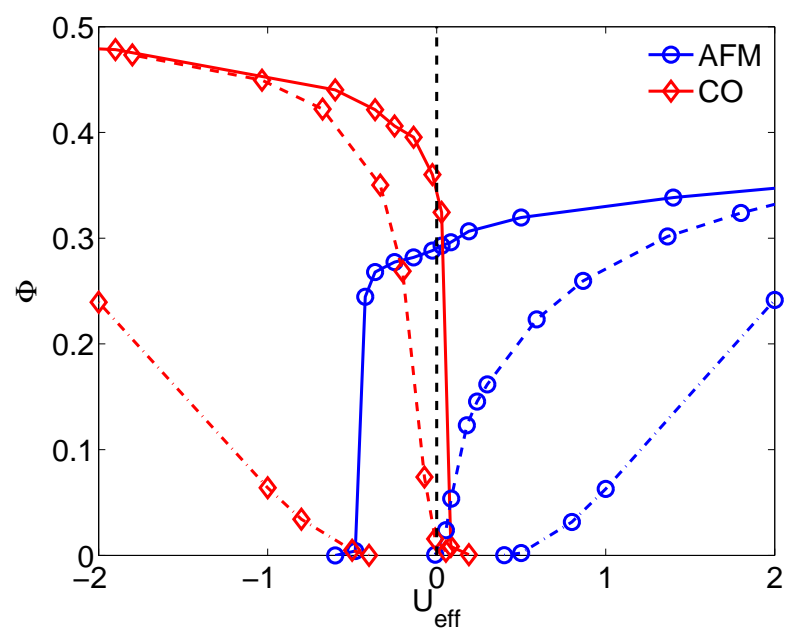

FIG. 7: (Color online) The expectation values $\Phi$ for a range of effective $U_{\text {eff }}=-2,2$ for fixed $U=3$ varying $g$. We show the results for $\omega_{0}=0.2$ (full line), $\omega_{0}=0.6$ (dashed line), and $\omega_{0} \rightarrow \infty$ (dot-dashed line).

as in mean field theory $\underline{48}$. The form of the transition is then symmetric with respect to $U_{\text {eff }}=0$. For finite $\omega_{0}$ when tuning $U_{\text {eff }}$ with $g$ the transition will be asymmetric and the order parameter is decreased much less when the effective interaction is close to 0 , as seen most pronounced for the case $\omega_{0}=0.2$ (full line). In the adiabatic limit, $\omega_{0} \rightarrow 0$, we always expect a discontinuous transition at zero temperature. For finite $\omega_{0}$ we have the competition of the Hubbard repulsion and phonon-induced attraction. The effect of latter enters at lower energies for smaller values of $\omega_{0}$. This might explain why the AFM order is more stable then. To establish $\mathrm{CO}$ as the ground state, it seems to be mainly necessary that $U \lesssim \lambda$, and the large $U$ at higher energies does not spoil this. Retardation effects seem to play hardly any role at half filling for these static orders.

We can conclude from this section that with generality the AFM-CO quantum phase transition occurs approximately when electron interaction parameter $U$ and phonon attraction $\lambda$ are equal. The behavior near the transition, e.g. the order parameter depends, however, very much on the the interaction strength as well as the phonon frequencies. Small phonon frequencies and large interactions lead to discontinuous behavior, whereas for large phonon frequencies the competing interactions lead to more cancellations, reduced order and much evidence for continuous transitions. Then there exists a point on the transition line $\lambda_{\mathrm{tc}} \simeq U$, separating continuous and discontinuous transitions. We find that the value $\lambda_{\text {tc }}$ increases with $\omega_{0}$. In the limiting case of $\omega_{0} \rightarrow \infty$, there are only continuous transitions such that $\lambda_{\text {tc }}=\infty$, whereas we expect that for $\omega_{0} \rightarrow 0$ there are only discontinuous transitions and $\lambda_{\mathrm{tc}}=0$. It would be of interest to explore how $\lambda_{\text {tc }}$ varies for finite $\omega_{0}$ as a function of temperature. As temperature tends to increase fluctuations and decrease order, the naive expectation would be that $\lambda_{\mathrm{tc}}$ increases with temperature.

\section{DIFFERENT CONTRIBUTIONS TO THE TOTAL ENERGY}

We have used the total ground state energy $E_{\text {tot }}$ to decide whether the AFM or CO state is the ground state. In this section we give details of the different contributions to $E_{\text {tot }}$ in (4), and their dependence on the electronphonon coupling for fixed $U$. Let us first remark generally on the energy of the ordered state in comparison with the normal $(\mathrm{N})$ state. In the half filled pure Hubbard model at weak coupling the AFM state has lower potential energy than the $\mathrm{N}$ state, but higher kinetic energy. At strong coupling the AFM has lower kinetic energy than the $\mathrm{N}$ state (exchange term), but higher potential energy than the $\mathrm{N}$ state (Mott insulator). In the pure Holstein model the CO state has a lower potential energy at weak and intermediate coupling and higher kinetic energy due to localization. At strong coupling when the $\mathrm{N}$ state is insulating (bipolaronic) the energy is lowered in the CO state due to lower kinetic energy (pair hopping).

In the situation with finite $U$ and $g$, we have a competition between the different terms. The AFM state will usually have smaller $E_{U}$, since double occupancy is lower, whereas the $\mathrm{CO}$ state possesses larger $E_{U}$ but contributions from $E_{g}$ lower the energy. However, we also have to take into account the contribution $E_{\mathrm{ph}}$, which is larger in the $\mathrm{CO}$ state.

In Fig. 8 we see the behavior of kinetic energy for the electrons $E_{\text {kin }}$ and oscillator energy of the phonons $E_{\mathrm{ph}}$, where the energy $\omega_{0} / 2$ for zero point motion was omitted.
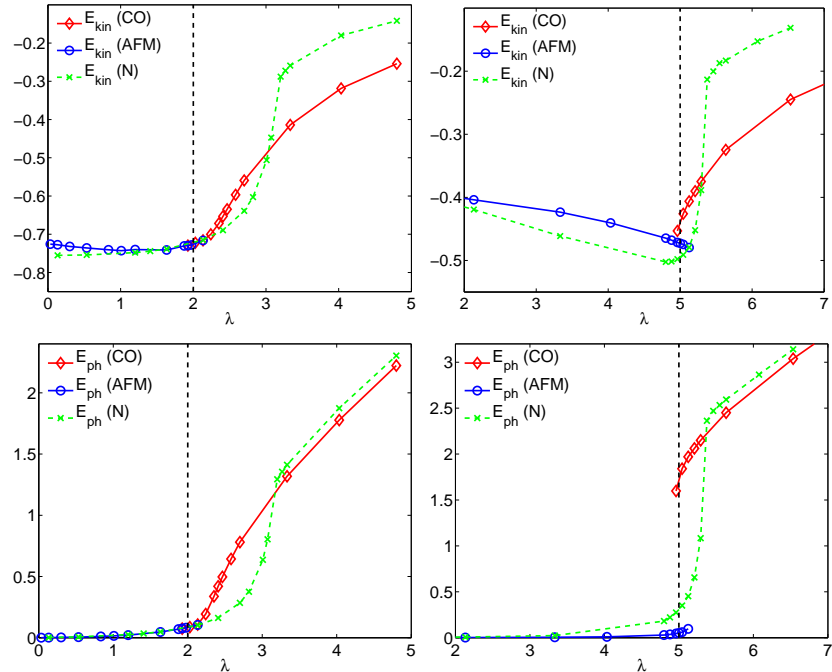

FIG. 8: (Color online) Comparison of the kinetic energy $E_{\text {kin }}$ and phonon energy $E_{\mathrm{ph}}$ for $U=2$ (left) and $U=5$ (right) as a function of $\lambda$ in $\mathrm{CO}, \mathrm{AFM}$ and $\mathrm{N}$ state.

We show the quantities for the values $U=2$ (left) and $U=5$ (right) as a function of $\lambda$ in $\mathrm{CO}, \mathrm{AFM}$ and $\mathrm{N}$ 
state. As a reference energy recall that for the free system $E_{\text {kin }}^{0} \simeq-0.849$. Similar to the pure Hubbard or Holstein model the electronic kinetic energy of the AFM and $\mathrm{CO}$ state is larger than the $\mathrm{N}$ (metallic) state, whilst the energy gain comes from the interaction energies, $E_{U}$ for the AFM state and $E_{g}$ for the CO state, see Fig. 9. both for $U=2$ and $U=5$. The situation is different for the BP state $(\lambda>3.1$ for $U=2$ and $\lambda>5.4$ for $U=5)$. Then the CO state wins through lower kinetic energy, whilst the interaction energy is lower in the BP state.

The phonon energy does not change much with $\lambda$ for values $\lambda<U$ in the $\mathrm{N}$ and AFM state, but increases rapidly for $\lambda>U$ both in the $\mathrm{N}$ and $\mathrm{CO}$ state. This is mainly due to the increase in potential energy $\omega_{0}^{2}\left\langle\hat{x}^{2}\right\rangle / 2$, as will be seen in detail in Section VI when we discuss $\left\langle\hat{x}^{2}\right\rangle$.
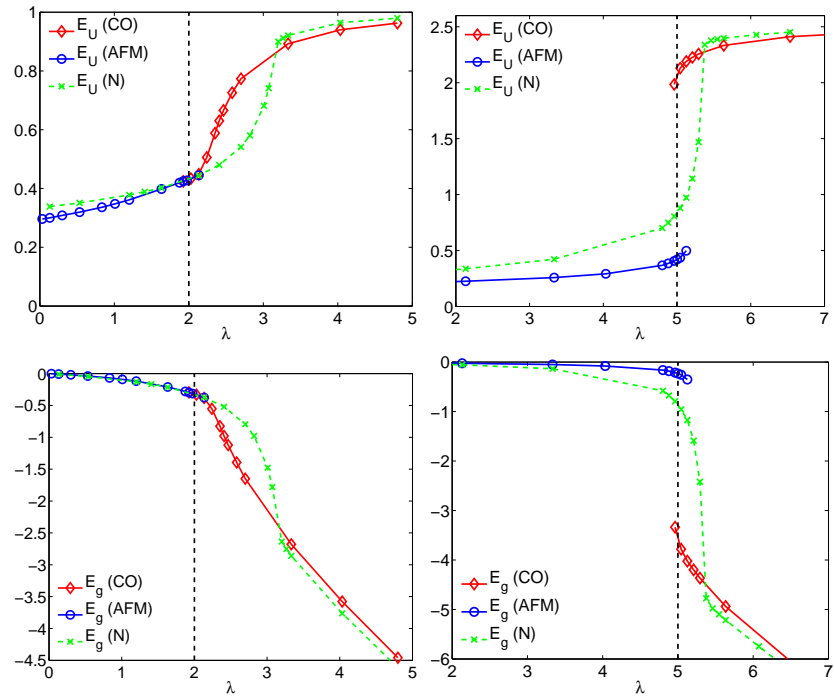

FIG. 9: (Color online) Comparison of the interaction energies $E_{U}$ and $E_{g}$ for $U=2$ (left) and $U=5$ (right) as a function of $\lambda$ in CO, AFM and $\mathrm{N}$ state.

The energy of the AFM state depends relatively little on the electron-phonon coupling, i.e. if the order is not destroyed, the phonons have a minor effect on the static properties. $E_{U}$ has a small value for weak electronphonon coupling and tends to $U / 2$ in the case of strong coupling for the $\mathrm{BP}$ and $\mathrm{CO}$ state. For weaker repulsion, $U=2$, the energies vary continuously with $\lambda$. However, for the stronger coupling case $U=5$ we can clearly see the discontinuities at the AFM-CO transition, $U \simeq \lambda$. $E_{\mathrm{ph}}$ jumps from a low value in the AFM state to a large value in the $\mathrm{CO}$ state. Due to the increase in double occupancy also $E_{U}$ increases suddenly at the transitions. Both of these energetically unfavorable contributions for the CO state are counterbalanced by the abrupt decrease of $E_{g}$, as the electron-phonon gives strong binding energy in the CO state. As a result the total energy is continuous at the transition, but shows kink.

For large $\lambda$ the interaction energy $E_{g}$ is proportional to $-\lambda$. For the $\mathrm{CO}$ state this can be understood through a factorization of the expectation value in $E_{g}$ in the electronic and phonon part. With equation (9) discussed later one finds then $E_{g} \rightarrow-4 \lambda \Phi_{\text {co }}^{2}$. Since in the ordered state $\Phi_{\text {co }}=1 / 2$ the behavior follows.

\section{QUASIPARTICLE PROPERTIES IN THE NORMAL STATE}

In a large part of the phase diagram in Fig. 1 near the AFM-CO transition, the system without symmetry breaking is in the $\mathrm{N}$ metallic state, which is a Fermi liquid. We can gain insight into the properties of the system, when we analyze the quasiparticle properties of the $\mathrm{N}$ state. The states with broken symmetry can then be viewed as instabilities of the Fermi liquid state. The renormalization factor $z$, which is related to the weight of the quasiparticle peak and for a $\boldsymbol{k}$-independent selfenergy to the inverse of the effective mass of the quasiparticles, $m^{*} / m_{0}=z^{-1}$. It can be calculated from the derivative of the self-energy as well as from the analysis of the NRG low energy excitations at the fixed point. From the latter procedure, one can also deduce a local effective quasiparticle interaction $U^{r}$ by comparing the energy of the lowest two-particle excitation energy $E_{p p}$ with the energy of two one-particle excitations $E_{p}, U^{r} \sim E_{p p}-2 E_{p}$. For details we refer to Ref. 36.49.

\section{A. Renormalized parameters as function of $\lambda$}

First we discuss how the quasiparticle weight $z$ varies with $\lambda$, when $U$ is held fixed. It is shown for various values of $U$ as a function of $\lambda$ in Fig. 10.

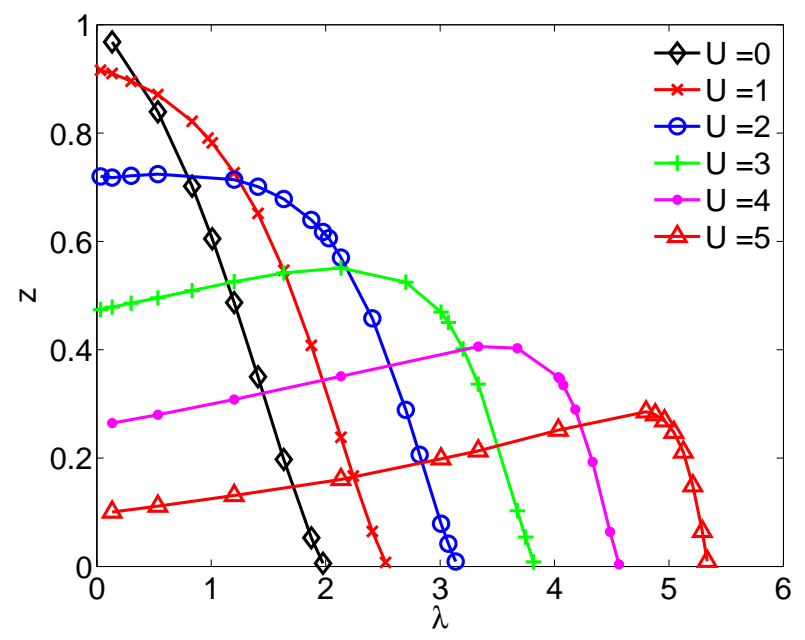

FIG. 10: (Color online) The $z$-factor for various $U$ as a function of $\lambda$.

We can identify different effects of the electron-phonon coupling. For $U=0$ and small values of $U$, increasing $\lambda$ 
leads to polaron formation and localization of the charge carriers, which results in a reduction of $z$ and a larger effective mass. Eventually, the metal-bipolaron transition is reached, where $z \rightarrow 0$. For larger values of $U$ the electrons are already renormalized for $\lambda=0$ due to the Coulomb interaction. The first effect of increasing the electron-phonon coupling is to reduce this effect and $z$ increases with $\lambda$. Note that the effect is substantially less than what would be expected for a pure Hubbard model with $U_{\text {eff }}$. The maximal value obtained occurs approximately when $\lambda \simeq U$, i.e. the renormalization effects cancel there to the largest extent leading to a least enhanced effective mass. Near the M-BP transition $m^{*} / m_{0}$ diverges as for the weak $U$ case. Apart from the approximate maximum no particular characteristic behavior is seen in $z$ near the AFM-CO transition, $U \simeq \lambda$.

As shown in Fig. 11 the effective quasiparticle interaction $U^{r}$ varies between positive and negative values depending on $U$ and $\lambda$. In the cases for finite $U$ it starts repulsive and goes to zero approximately where $U_{\text {eff }}$ does. Then it becomes negative such that there is an effective attraction between quasiparticle excitations. There is a slight shift towards $\lambda>U$ for this sign change to occur for larger values of $U$. This is in line with the earlier observation that the $\mathrm{CO}$ state becomes the ground state when $\lambda>U$.

The change of sign of $U^{r}$ when $U \sim \lambda$ can be related to the maximum found for $z$ at this point in Fig 10. At $U \sim \lambda, U^{r} \sim 0$, the quasiparticles are effectively noninteracting. As $\lambda$ is varied from this point, both $\left|U_{\text {eff }}\right|$ and $\left|U_{r}\right|$ increase which causes a further renormalization of the quasiparticles and a reduction in the value of $z$. The decrease in $z$ from $U^{r}=0$ occurs irrespective of whether $U^{r}<0$, as in the pure Holstein model, or $U^{r}>0$, as in the Hubbard model.

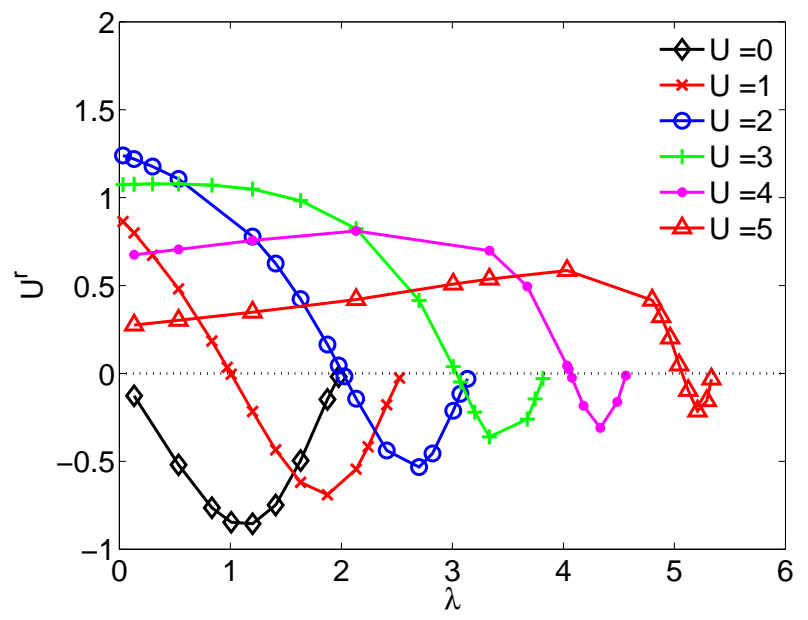

FIG. 11: The effective quasiparticle interaction $U^{r}$ for various $U$ as a function of $\lambda$.

Taking the viewpoint of instabilities of the Fermi liquid, one can infer that the ground state, AFM or CO, is determined by whether the low energy quasiparticles interact attractively or repulsively. The sign change in turn occurs when the bare parameters $U$, and $\lambda$ on high energies are equal. One might have expected that in reducing the energy scale $\omega$ down to the phonon frequency $\omega_{0}$ that the main retardation effects would renormalize $U$ to some effective value $\bar{U}$, where $\bar{U}$ would be of the order of $U^{r}$ in the pure Hubbard model, which is such that $U^{r} \ll U$. The attractive term induced by the phonons would then contribute for $\omega \ll \omega_{0}$. The change of sign of the quasiparticle interaction would then be expected to occur when $\bar{U} \sim \lambda$, which would correspond to a much smaller value of $\lambda$ than $\lambda \sim U$. The fact that the transition and the change of sign of $U^{r}$ are found to occur when $U \sim \lambda$ indicates that $U$ term and the $\lambda$ term are renormalized in a similar way as the energy scale is reduced. As noted before, however, when discussing the order parameter, the system cannot be described simply by an effective Hubbard model and both the electronic interaction $U$ and $\lambda$ play a role in determining the properties of the system in a certain phase. For instance, the $\mathrm{CO}$ order parameter in Fig. 4 corresponding to $U_{\text {eff }} \simeq-0.01$ is $\Phi_{\mathrm{co}} \simeq 0.4$, but the result would be close to zero for the pure Hubbard model with this interaction on all energy scales.

When $\lambda$ exceeds a certain value $\left|U^{r}\right|$ decreases again, and we can also see that not only $z$ but also the effective quasiparticle interaction $U^{r}$ goes to zero at the M-BP transition. It is of interest to study the combined quantity $U^{r} / z=U^{r} m^{*}$, which is plotted in Fig. 12,

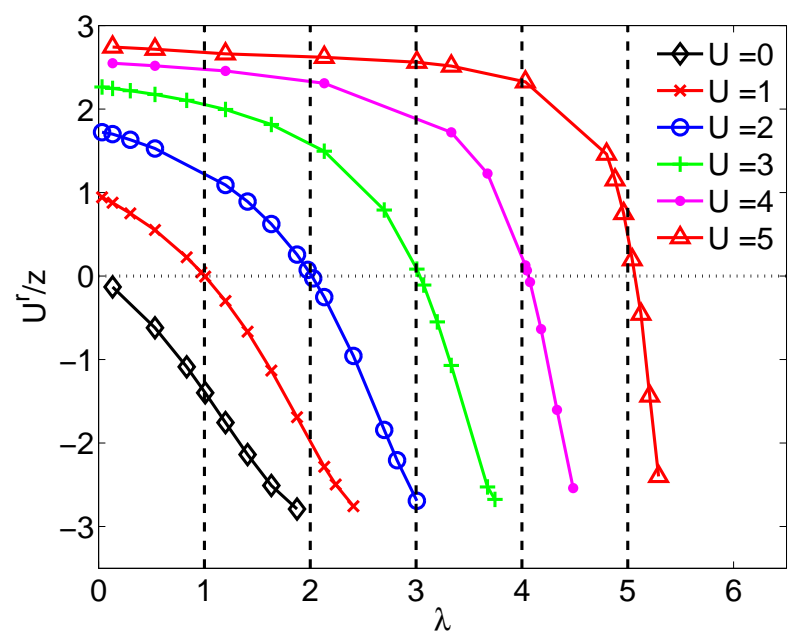

FIG. 12: The quantity $U^{r} / z$ for various $U$ as a function of $\lambda$.

This product of the effective quasiparticle interaction and effective mass takes into account the aspect of the localization tendency of the quasiparticles as well as their residual interaction. We can see that it shows a more universal behavior for the different cases. It decreases monotonically as a function of $\lambda$ for the given values of $U$. Close to the M-BP transition in all cases $U^{r} m^{*}$ tends to a value between 2.5 and 3 . One is therefore tempted to identify this as the relevant quantity for an instability of 
the metallic state, such that the M-BP transition occurs.

\section{B. Renormalized parameters as function of $U$}

Similarly, we can also study the behavior of the quasiparticle properties for fixed $\lambda$ as a function of $U$. The quasiparticle weight $z$ is shown for various values of $\lambda$ as a function of $U$ in Fig. 13 .

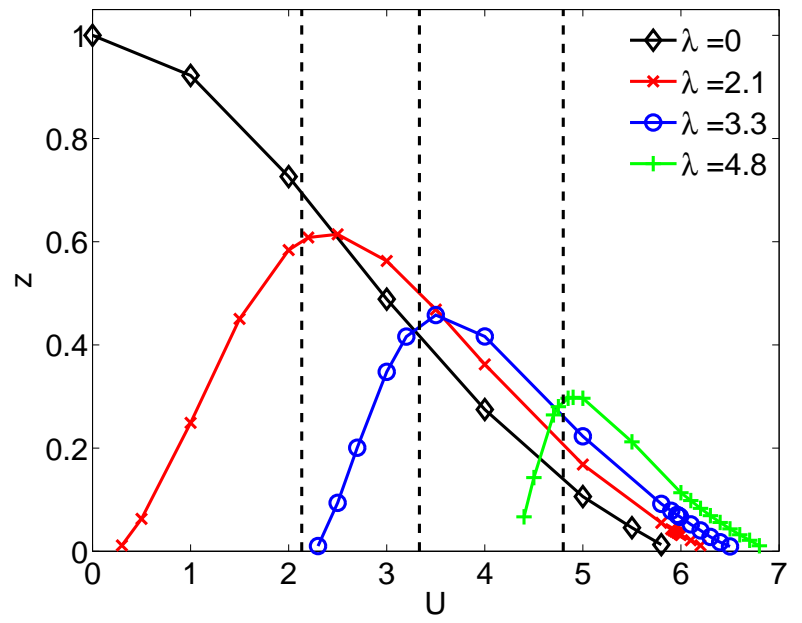

FIG. 13: The $z$-factor for various $\lambda$ as a function of $U$.

For $\lambda=0, z$ is monotonically driven to zero when increasing $U$ to the Mott transition. For finite values of $\lambda$ increasing $U$ leads to an increase in $z$ when we come from the bipolaronic state and thus to a de-renormalization as $\left|U_{\text {eff }}\right|$ decreases. Similar to the case observed above but more pronounced, one finds $z$ to be approximately maximal near the AFM-CO transition, $U \simeq \lambda$, i.e. when $U_{\text {eff }}$ as well as $U^{r}$ are close to zero. Different renormalization effects have then canceled to maximal extent, leaving relatively weakly renormalized, nearly noninteracting quasiparticles. Again it is of interest to study the quantity $U^{r} m^{*}$, which is plotted in Fig. 14.

As noted before it goes to zero where $U_{\text {eff }}$ does. Then it changes sign and increases with $U$ as long as the system is in the metallic state. Close to the Mott transition it approaches a value 2.5-2.7 which is approximately the same for different values of $\lambda$. It seems therefore that for this metal insulator transition a universal value determines when it occurs.

\section{PROPERTIES OF THE PHONONS}

So far we have studied the CO-AFM transition via the electronic properties, which where greatly influenced by the interaction with the bosonic modes. In turn the properties of the local harmonic oscillator modes are also modified by the coupling to the electronic degrees of the freedom, depending on the different coupling strengths.

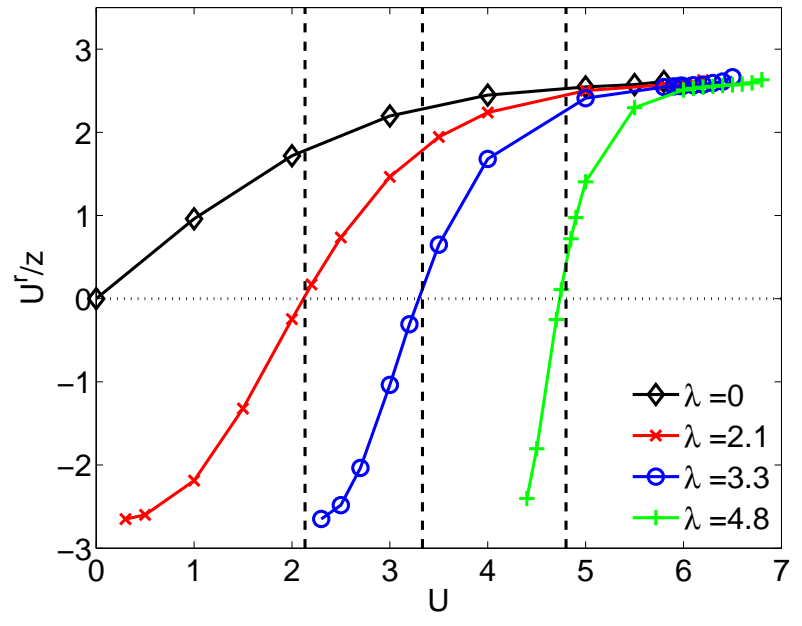

FIG. 14: The quantity $U^{r} / z$ for various $\lambda$ as a function of $U$.

First we consider the phonon number expectation value $n_{\mathrm{ph}}=\left\langle b^{\dagger} b\right\rangle$. It is expected to increase in the CO (BP) state as a high probability of local double occupation leads to a charge redistribution and thus a (temporal) displacement of the lattice ions. This means that phonons become excited, multiplied with $\omega_{0}$ this gives the energetic contribution $E_{\mathrm{ph}}=\omega_{0} n_{\mathrm{ph}}$ This was discussed in Section IV] in Fig. 8 for $U=2$ and $U=5$ as a function of $\lambda$.

We find that the low value for $n_{\mathrm{ph}}$ in the AFM state increases quite slowly as the coupling strength is increased. Once the transition to the CO state has occurred $n_{\mathrm{ph}}$ increases substantially. The expectation values connect continuously, but increase rapidly for larger $\lambda$. The value for the normal state lies below the $\mathrm{CO}$ result but increases rapidly for $\lambda \simeq 3$, where the metal-bipolaron transition occurs. This behavior can be compared with the result for larger $U=5$, which is shown in Fig. 8 (bottom right). We can see that $n_{\mathrm{ph}}$, being small in the AFM state, remains nearly unaltered when the coupling is increased. The large $U$ and strong AFM order suppresses local charge fluctuations. The value increases rapidly near $U_{\text {eff }}=0$ in the $\mathrm{CO}$ state. The expectation value changes therefore discontinuously at the transition. The behavior in the normal state is similar to the earlier case and the metal-bipolaron transition $(\lambda \simeq 5.3)$, which is also discontinuous for these parameters.

The charge order, which we have characterized by $\Phi_{\mathrm{co}}$, can also be seen directly in the displacement expectation value on the $A$-sublattice $\left\langle\hat{x}_{A}\right\rangle=\left\langle b_{A}+b_{A}^{\dagger}\right\rangle / \sqrt{2 \omega_{0}} \equiv\langle x\rangle$. This value is always zero in the AFM and $\mathrm{N}$ state but finite once the CO symmetry is broken. In Fig. 15 it is plotted for various values of $U$ as a function of $\lambda$.

We can see that similar to the behavior of the order parameter $\langle x\rangle$ increases close to the transition, and more rapidly for larger $U$. In its dependence on the coupling strength, it appears to be very similar to the order parameter (Figs. 2] and 4). In fact, one can show that they 


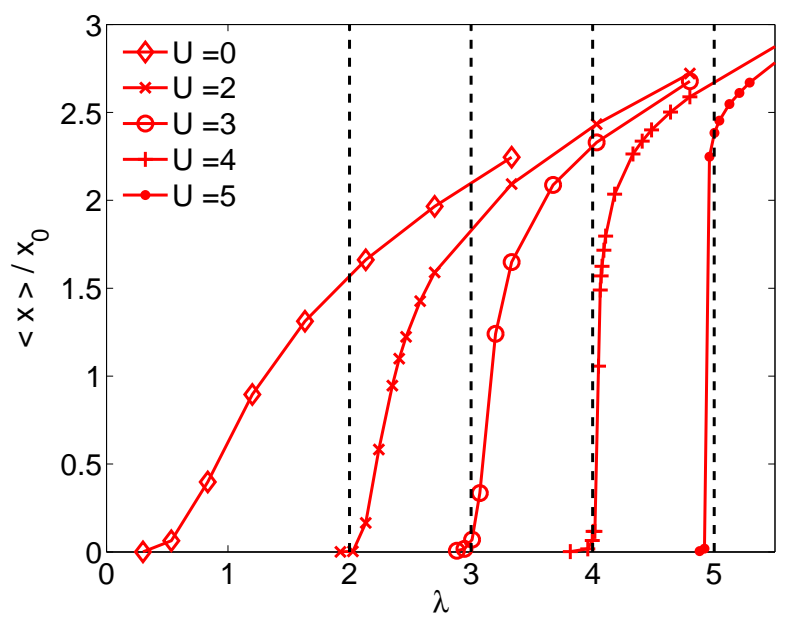

FIG. 15: (Color online) The expectation values $\langle x\rangle$ for various values of $U$ as a function of $\lambda$ for $\omega_{0}=0.6$ in the CO state.

are directly related by an exact identity,

$$
\langle x\rangle=-\frac{2 \sqrt{\lambda}}{\omega_{0}} \Phi_{\mathrm{co}},
$$

which can be derived by considering an additional term $H_{c}^{i}=\omega_{0} c\left(b_{i}+b_{i}^{\dagger}\right)$ in the Hamiltonian and calculating the derivative with respect to $c$. The numerical values for the left and right hand side of equation (9) agree very well. We can see that the slope at the transition increases with $U$. At large $U$, or very small $\omega_{0}$, there is a very sharp transition as seen for the case $U=5$.

The effect of the strong electron lattice coupling can be seen in the displacement fluctuations $\left\langle\hat{x}^{2}\right\rangle$, which are plotted in Fig. 16 for $U=2$ and $U=5$ and a range of $\lambda$. The behavior is reminiscent of the phonon expectation value $n_{\mathrm{ph}}$, where a continuous rise near the transition is visible for weaker coupling and a discontinuity from the AFM to the CO state of the stronger coupling case. Here also the normal BP state possesses a larger value than the $\mathrm{CO}$ state. The close comparison with the results for $n_{\mathrm{ph}}$ shows that for large coupling in the $\mathrm{BP}$ and $\mathrm{CO}$ state $x_{0}^{2}\left(1+4 n_{\mathrm{ph}}\right) / 2$ gives a good fit. This result can be derived by taking $n_{i, \uparrow}=n_{i, \downarrow}=1$, and performing a displaced oscillator transformation to new phonon operators, $a, a^{\dagger}$, $a^{(\dagger)}=b^{(\dagger)}+g / \omega_{0}$. The ground state $|g \mathrm{~s}\rangle$ then corresponds to the state $a|\mathrm{gs}\rangle=0$, and in this state $\left\langle b^{\dagger} b\right\rangle=g^{2} / \omega_{0}^{2}=$ $n_{\mathrm{ph}}$, and $\left\langle\hat{x}^{2}\right\rangle=x_{0}^{2}\left(1+4 g^{2} / \omega_{0}^{2}\right) / 2$, giving the required result. The state $|\mathrm{gs}\rangle$ in the original basis corresponds to the coherent state $\sum_{n} \frac{\alpha^{n}}{\sqrt{n !}}|n\rangle\left(b^{\dagger} b|n\rangle=n|n\rangle\right)$, with $\alpha=-\sqrt{n_{\mathrm{ph}}}$, and the result can alternatively be derived by taking the expectation value of $\hat{x}^{2}$ in this state. The decoupled oscillator state is an eigenstate $|n\rangle$, but when strongly coupled to the electronic system the nature of the state changes to the coherent ground state due to the displacement of the oscillator.

If we multiply $\left\langle\hat{x}^{2}\right\rangle$ by $\omega_{0}^{2} / 2$ we obtain the potential energy of the harmonic oscillator. The comparison with
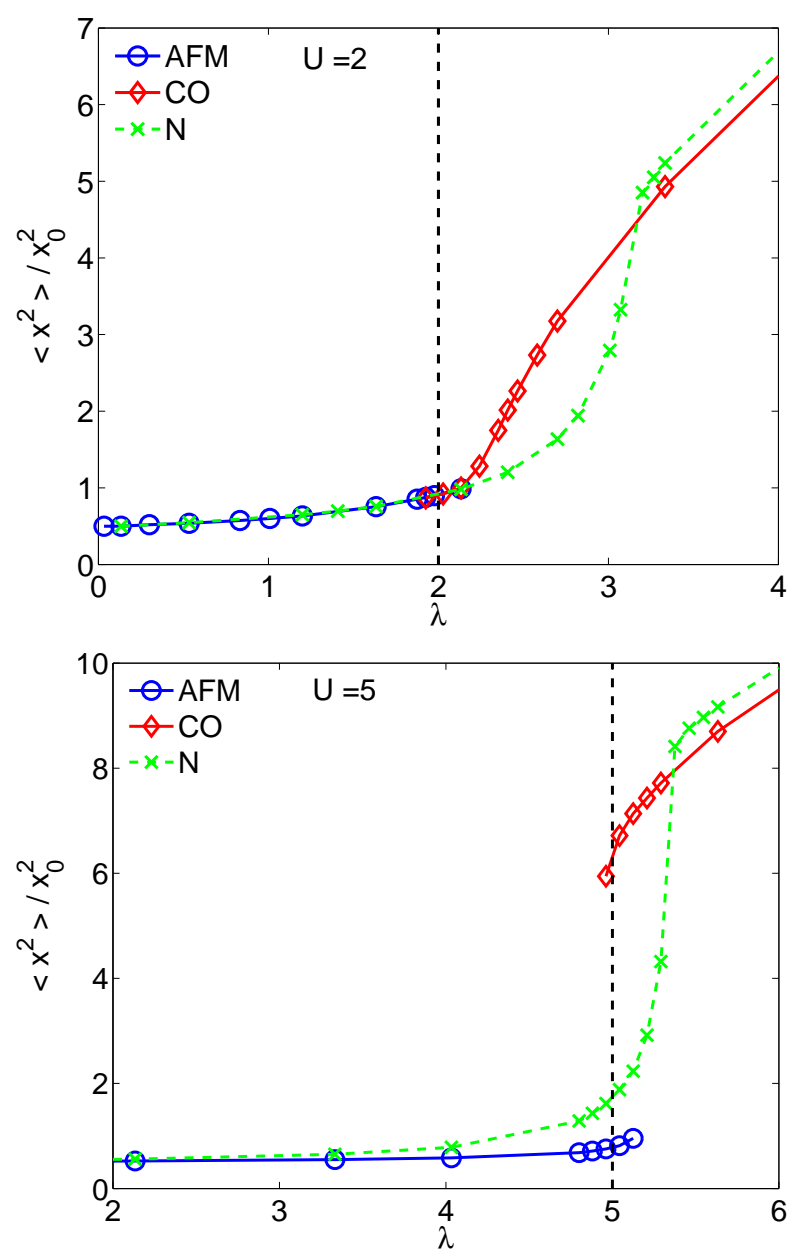

FIG. 16: (Color online) The displacement fluctuations $\left\langle\hat{x}^{2}\right\rangle$ for $U=2,5$ as a function of $\lambda$ for $\omega_{0}=0.6$ for the AFM, CO and normal state.

$E_{\mathrm{ph}}$ shows then that most of the phonon energy is in the potential energy due to the charge redistribution, and only small proportion in the kinetic energy of the oscillator.

The real lattice fluctuations are large in the $\mathrm{BP}$ state where the local occupancy changes from double to zero, but $\langle\hat{x}\rangle=1$. A measure of these fluctuations is the quantity $\Delta x^{2}=\left\langle(\hat{x}-\langle\hat{x}\rangle)^{2}\right\rangle=\left\langle\hat{x}^{2}\right\rangle-\langle\hat{x}\rangle^{2}$. This is a much smaller quantity than $\left\langle\hat{x}^{2}\right\rangle$ in the CO state and only large near the transition, as can be seen in Fig. 17. In the uncoupled state $\Delta x^{2} / x_{0}^{2}=\left\langle\hat{x}^{2}\right\rangle / x_{0}^{2}=1 / 2$. In the displaced oscillator (coherent) state, which describes the strong coupling situation, $\langle\hat{x}\rangle^{2}=2 x_{0}^{2} g^{2} / \omega_{0}^{2}$, so combining this with the expression derived earlier for $\left\langle\hat{x}^{2}\right\rangle$, one again finds $\Delta x^{2} / x_{0}^{2}=1 / 2$. Both limiting cases can be found in Fig. 17, where $\Delta x^{2}$ increases with $\lambda$ in the AFM state. It then falls again to $1 / 2$, when the system is strongly ordered. So the "lattice fluctuations" are largest at the transition.

It is possible to use the density matrix approach in the NRG ${ }^{50}$ together with the real space harmonic oscil- 


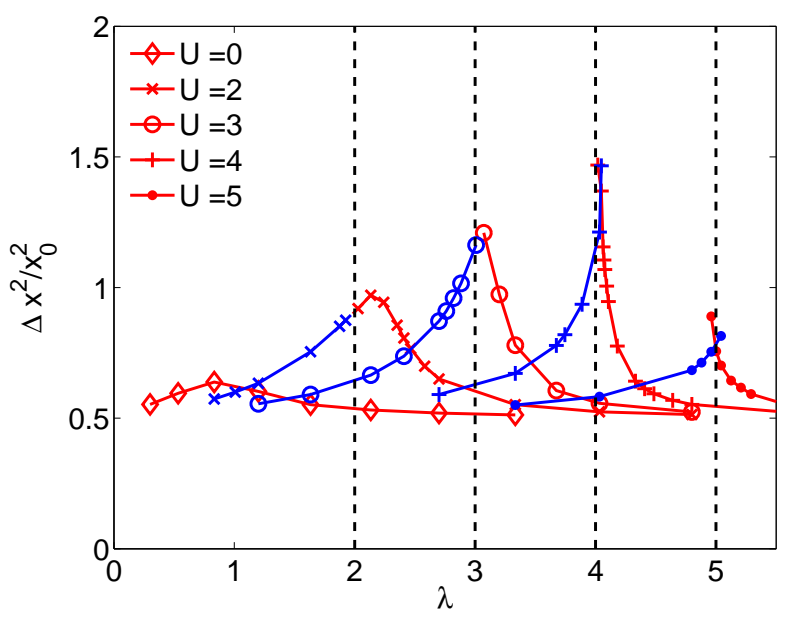

FIG. 17: (Color online) The expectation values $\Delta x^{2}$ for various values of $U$ as a function of $\lambda$ for $\omega_{0}=0.6$ in the AFM state for $\lambda<U$ and in the CO state for $\lambda>U$.

lator eigenfunctions to compute the oscillator displacement probability function $P(x)$, which gives further insight into the behavior of the phonons. Details for this method have been presented elsewhere ${ }^{51}$. The moments of this distribution function, $\left\langle\hat{x}^{m}\right\rangle=\int \mathrm{d} x P(x) x^{m}$, can be calculated from $P(x)$ and are in agreement with the value determined from the groundstate expectation values.

\section{SPECTRAL PROPERTIES}

In this section we turn to the excitation spectra of the coupled electron-phonon system. We first consider the electronic local lattice Green's function $G_{\alpha, \sigma}(\omega)$, which is given by the momentum sum of the diagonal element of (2), and its spectral function $\rho_{\alpha, \sigma}(\omega)=-\operatorname{Im} G_{\alpha, \sigma}(\omega) / \pi$. In these calculations the sublattice self-energies $\Sigma_{\alpha, \sigma}(\omega)$ enter, which are calculated according to Eq. (B1) following Bulla et al .52 . We will comment on complications which can arise in appendix B $\rho_{\alpha, \sigma}(\omega)$ is of special interest in studying the broken symmetry behavior. In the normal state the Green's functions are the same for the sublattices as well as the spin projections. For the cases with symmetry breaking, $\mathrm{CO}$ and AFM, these function will differ, i.e. for the $\mathrm{CO}$ case the sublattice Green's functions differ and for the AFM case the different spin projections. We focus on the $A$-sublattice majority spin spectral function $\rho_{A, \uparrow}(\omega)$. Note that at half filling the spectra for minority spin in the AFM case, $\rho_{A, \downarrow}(\omega)$, and for the $B$-lattice for the charge order, $\rho_{B, \uparrow}(\omega)$, can be obtained from $\omega \rightarrow-\omega$. In order to calculate the full electron Green's function one has to put the different sublattice Green's functions together $\underline{36}$.

\section{A. Electron spectra}

We first consider the electronic spectral functions near the transition for the cases of $U=2$ and $U=5$. In Fig. 18 we plot the $\mathrm{N}$ state spectral function in comparison with the corresponding symmetry broken one. We have included a $\mathrm{N}$ state spectrum for $\lambda \rightarrow 0$ in order to first see the effect of the phonons and associated modification in the normal state.
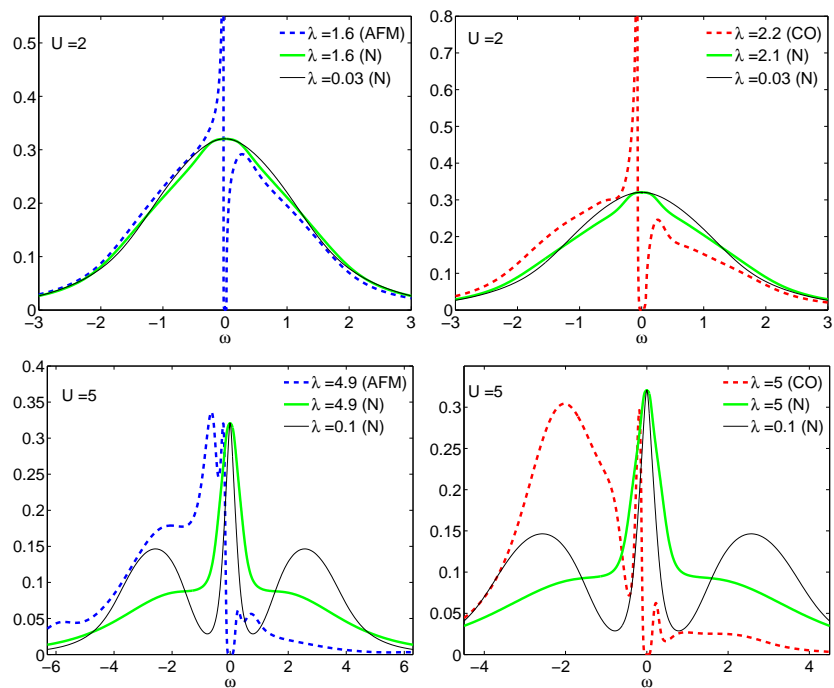

FIG. 18: (Color online) The local $A$-lattice spectral functions in comparison for $U=2$ (upper panel) and $U=5$ (lower panel). Left: Comparison of $\mathrm{N}$ state spectrum with AFM majority spin $\rho_{A, \uparrow}(\omega)$ near the transition. Right: Comparison of $\mathrm{N}$ state spectrum with $\mathrm{CO} A$-site $\rho_{A, \uparrow}(\omega)$ near the transition.

For $U=2$ in the upper part of the figure we see that close to the transition the $\mathrm{N}$ state spectrum deviates little from the $\lambda \rightarrow 0$ situation, with only a small extra renormalization of the low energy excitations $[z(\lambda=0)=0.73$ and $z(\lambda=2)=0.57$; the metal bipolaron transition with $z \rightarrow 0$ occurs for a larger $\lambda \simeq 3$. The imaginary part of the electronic self-energy due to electron phonon scattering becomes finite when $|\omega|>\omega_{0}^{r}$, where $\omega_{0}^{r}$ is the renormalized phonon frequency (see Sec. VIIB).

For the AFM and CO symmetry broken state we can see characteristics of the weak coupling instability at the Fermi surface $(\omega=0)$ in the sublattice spectral function. It fits well to the mean field description, where a square root divergence is found below the gap $\frac{35}{2}$ The higher energy parts are little modified for the case of $U=2$ apart from the broadening of the band edges, but no features which can be attributed to the phonons can be identified.

In the lower panel of Fig. 18 we can see the situation for $U=5$. For $\lambda \rightarrow 0$ we have the well-known three peak structure with lower and upper Hubbard band and a quasiparticle peak in the $\mathrm{N}$ state. For $\lambda \simeq 5$ the quasiparticle structure is still visible, but the Hubbard peaks have been modified to high energy shoulders and cannot really 
be recognized any more. The effect is as if the effective electron-electron interaction is screened by the phonons. The quasiparticle weight becomes larger in this regime on increasing $\lambda[z(\lambda=0)=0.1$ and $z(\lambda=5)=0.27$, see also Fig. 11. The low energy features of the spectra with symmetry breaking look similar. Directly above and below the spectral gap one sees pronounced peaks with larger weight for the one below the gap. At higher energies the spectra look different in both situations. Here both features from the large $U$ as well as from higher order polaronic behavior can play a role. When interpreting the spectra one has to take into account the broadening and the limited energy resolution of the NRG at higher energies, which limit the accuracy. The AFM state with strong electron phonon coupling seems to show polaronic behavior at multiples of $\omega_{0}$ with decreasing weight. The charge ordered spectrum shows a principal peak at a position, which is a bit less than the fully polarized mean field shift $U n_{-\sigma}^{A}-2 \lambda \Phi_{\text {co }} \approx 2.5$. The large couplings for this case play a role on different energy scales. One may note that for the given parameters the order parameters are rather large, $\Phi \approx 0.4$ (see Fig. 4 ), whereas the spectral gap is relatively small.

To study the behavior of the spectral functions at different couplings we give various plots in Fig. [19] On the left hand side we keep $U=2$ fixed and show (from top to bottom) the spectra for N, AFM and CO state for various $\lambda$. On the right hand side $U=5$ is kept fixed.

The top panel shows the metal to bipolaron transition when $\lambda$ is increased. One can see the strong narrowing of the quasiparticle band near the transition, which is accompanied by $z \rightarrow 0$. A spectral gap develops when $\lambda$ exceeds a critical coupling. The details of the spectral functions have been analyzed by Koller et al. ${ }^{17}$. In the AFM case in the middle we can see for the weaker coupling case how, on increasing $\lambda$, the AFM order and magnitude of the spectral gap decreases. The electron phonon coupling is effective here in screening the repulsive $U$-term. No polaronic features can be identified in the spectra as the coupling is fairly weak. At stronger coupling, the AFM state is hardly affected for a range of $\lambda$. When approaching the transition we find visible modifications of the spectral functions including a reduction of the spectral gap and polaronic peaks.

In the bottom part the spectra in the CO state can be seen. Near the transition the spectra differ for the weak and strong coupling case as discussed before, but when $\lambda$ exceeds $U$ by a certain amount the spectra look very similar, and the different $U$ term is not directly visible anymore. As noted before the main peak for the sublattice spectra is located near the mean field shift and its position moves linearly with $\lambda$ as expected. There is a pronounced quasiparticle peak near the transition which becomes suppressed for larger values of $\lambda$. This suppression can be partly due to the broadening in the NRG procedure as discussed in detail for superconducting solutions $\frac{48}{}$.
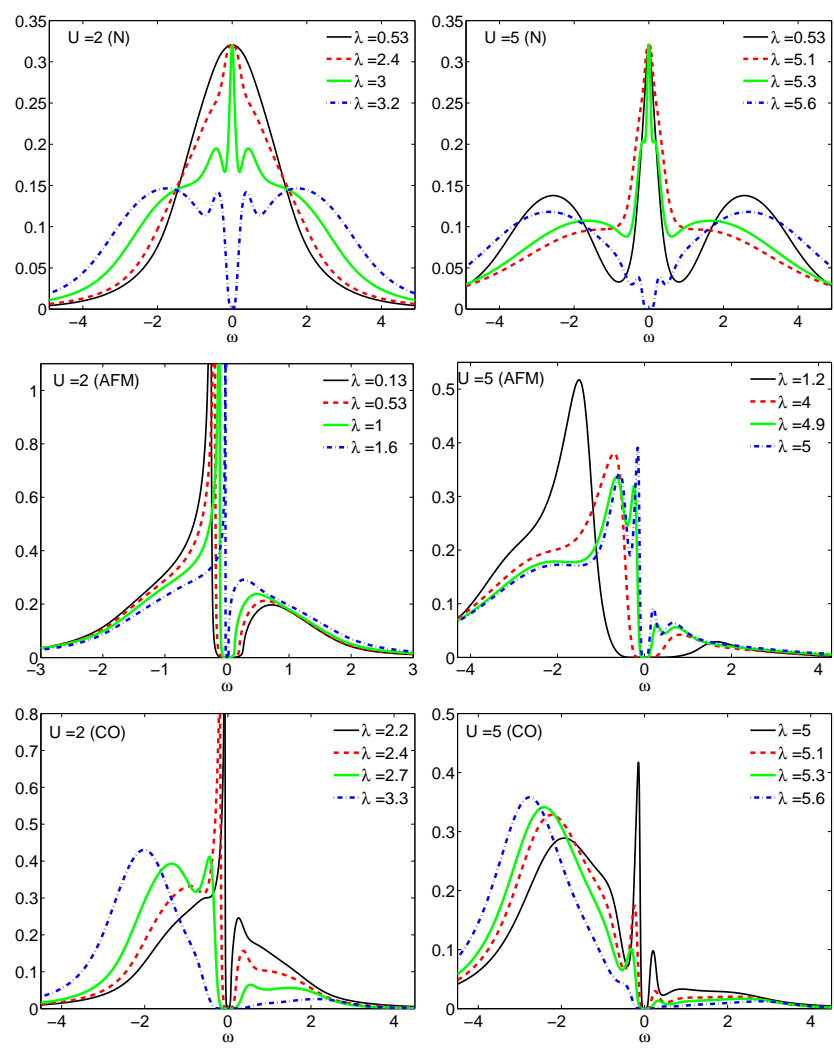

FIG. 19: (Color online) The local $A$-lattice spectral functions in comparison for $U=2$ (left) and $U=5$ (right) for various $\lambda$ and $\omega_{0}=0.6$. Top panel: $\mathrm{N}$ state spectrum. Middle panel: AFM majority spin $\rho_{A, \uparrow}(\omega)$. Lower panel: CO $A$-site $\rho_{A, \uparrow}(\omega)$.

\section{B. Phonon spectra}

In this section we study the spectral properties of the phonons. From these we can find out how the excitations of the bosonic sector are modified through the interaction with the electronic system. Especially near a transition a strong phonon softening can be indicative of a lattice modification or instability. We consider the function

$$
B(\omega)=\left\langle\left\langle b ; b^{\dagger}\right\rangle\right\rangle_{\omega},
$$

which can be calculated in the NRG from the matrix elements and excitations. The spectral function, $\rho_{b}(\omega)=$ $-\operatorname{Im} B(\omega) / \pi$, has the properties at $T=0$,

$$
\int_{-\infty}^{\infty} \mathrm{d} \omega \rho_{b}(\omega)=1, \quad \int_{-\infty}^{0} \mathrm{~d} \omega \rho_{b}(\omega)=-n_{\mathrm{ph}},
$$

and the free propagator has the form

$$
B^{0}(\omega)=\frac{1}{\omega^{+}-\omega_{0}} .
$$

We define a phonon self-energy $\Sigma_{\mathrm{ph}}(\omega)$ the full propagator reads

$$
B(\omega)=\frac{1}{\omega^{+}-\omega_{0}-\Sigma_{\mathrm{ph}}(\omega)} .
$$


For a decoupled electron-phonon system $\Sigma_{\mathrm{ph}}(\omega)=0$ such that $\rho_{b}(\omega)$ is a delta function peaked at $\omega_{0}$. In the strongly interacting system the mode can be renormalized to $\omega_{0}^{r}$ and broadened, however, no $\boldsymbol{q}$-dependence develops in the infinite dimensional model.

Again we focus on the cases with fixed $U=2$ and $U=5$ for variable $\lambda$, and we compare the results from the $\mathrm{N}$ state with the ordered state. In Fig. 20 we plot in the upper part the results for the spectral function of the phonons $\rho_{b}(\omega)$ for $U=2$ for the $\mathrm{N}$ state (left) and the ordered states (right) and the lower part for $U=5$.

When the electron and phonon systems are weakly coupled the expected delta-function for $\rho_{b}(\omega)$ is found. When $\lambda$ is increased the phonon mode is substantially renormalized and softens, most markedly at the M-BP transition for $\lambda \simeq 3$ where $\omega_{0}^{r} \rightarrow 0$. The negative spectral weight for $\omega$, which builds up there, is directly related to the phonon expectation value $n_{\mathrm{ph}}$. In the $\mathrm{BP}$ state it is not resolved any more. The phonon mode then hardens back to $\omega_{0}$. Apart from the softening we also find a broadening of the phonon spectrum, when the system is strongly coupled. The behavior for $U=5$ is similar to the $U=2$. A more detailed discussion can be found in Ref. 17.
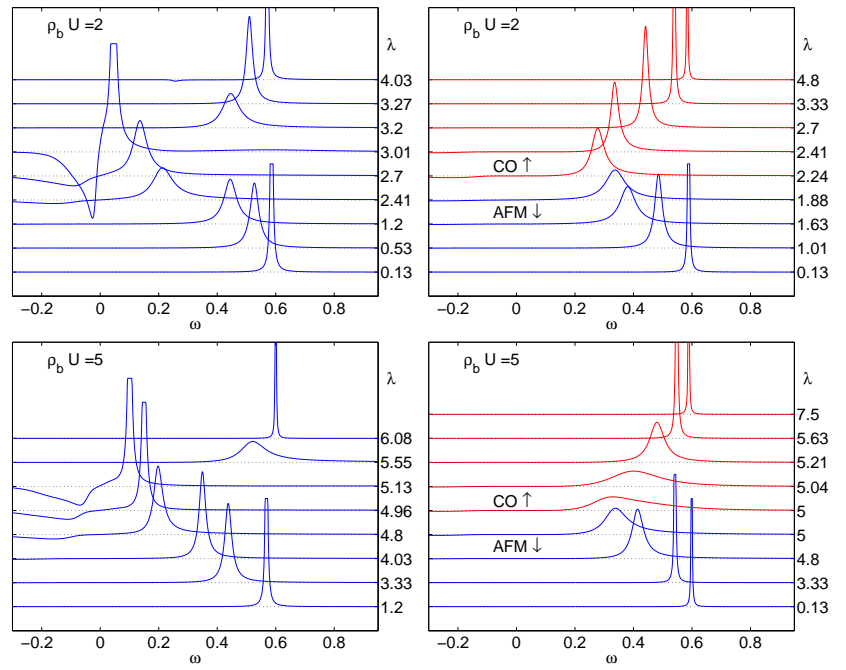

FIG. 20: (Color online) The local phonon spectral functions $\rho_{b}(\omega)$ in comparison for $U=2$ (upper panel) and $U=5$ (lower panel). Left: $\mathrm{N}$ state. Right: AFM state (smaller values of $\lambda$ and CO state for the larger values of $\lambda$.

In the right hand side panels of Fig. 20 the corresponding behavior of $\rho_{b}(\omega)$ is shown in the states with broken symmetry. The results for $\lambda<U$ correspond to the AFM phase and the ones for $\lambda>U$ to the $\mathrm{CO}$ phase. Comparing the metal-bipolaron transition to the AFM-CO transition we find that the effect of the phonon softening is much reduced. For $U=2$ we can see a visible effect that the oscillator mode is renormalized to $\omega_{0}^{r} \simeq 0.27$ and broadened. The effect is comparable to the normal state for the same values of $U$ and $\lambda$, but not as strong as at the M-BP transition. The softening is of similar magnitude in the stronger coupling case $U=5$. There a significant broadening of the phonon mode visible near the transition due to the large coupling of the phonons to the electronic system. When the system is well in the ordered state, AFM or CO, the phonon dynamics is little modified by the electronic system and a nearly free phonon mode is observed both for large $U \gg \lambda$ in the AFM state and $U \ll \lambda$ in the $\mathrm{CO}$ state. The sum rules for $\rho_{b}(\omega)$ given in equation (11) are satisfied to within a few percent for smaller values of $\lambda$. For larger $\lambda$ in the $\mathrm{BP}$ and $\mathrm{CO}$ state the sum rules are not well satisfied due to reasons discussed in Ref. 17.

\section{CONCLUSIONS}

In this study of the competing interactions in the Holstein-Hubbard model we have examined the transitions to both AFM and CO, both in the weak and strong coupling regimes. To lowest order, the effective frequency dependent interaction $U_{\text {eff }}(\omega)$ between the electrons is given by

$$
U_{\mathrm{eff}}(\omega)=U+\frac{2 g^{2} \omega_{0}}{\omega^{2}-\omega_{0}^{2}},
$$

the second retarded term arising from phonon exchange. On the lowest energy scale $\omega=0, U_{\text {eff }}(0)=U-\lambda$ $\left(\lambda=2 g^{2} / \omega_{0}\right)$, and the sign of this interaction depends on the relative strength of $U$ and $\lambda$. In studying the competition between AFM and CO, it is not surprising to find that the transition between these states occurs when $U \sim \lambda$, as $U_{\text {eff }}(0)>0$ favors AFM and $U_{\text {eff }}(0)<0$, the $\mathrm{CO}$ state. What is a surprising result of this study is that this condition still has some validity in the strong coupling regime, when both $U$ and $\lambda$ are large, as the transition is still found to occur when $U \sim \lambda$. We find, however, the nature of the transition does depend on the strength of the couplings, and also the phonon frequency $\omega_{0}$. The transition is found to be continuous for weak couplings, and a high phonon frequency $\omega_{0}$, but becomes discontinuous in the strong coupling regime, and for smaller values of $\omega_{0}$.

To gain further insight into this result, we have looked in detail at the quasiparticle excitations in the normal state. We have calculated both the quasiparticle weights $z$ and the effective local quasiparticle interaction $U^{r}$. We find that the local quasiparticle interaction $U^{r}$ changes sign when $U \sim \lambda$, just in the region where the AFM-CO transition occurs; this is consistent with the interpretation of the transition as due to a Fermi liquid instability. Though the interaction between the quasiparticle goes to zero at $U \sim \lambda$, the quasiparticles may still be quite significantly renormalized. For example, for $U=\lambda=5$ we find $z \simeq 0.3$. The fact that the local quasiparticle interaction goes to zero in the region $U \sim \lambda$, corresponding to $U_{\text {eff }}(0)=0$, suggests that the two terms contributing to $U_{\text {eff }}(\omega)$, are renormalized on the very low energy scale in a similar way. This is somewhat surprising, as in 
considering similar competing interactions in the case of superconductivity, it is generally assumed that the dominant renormalization is of the Coulomb term so that it does not overwhelm the attractive term from phonon exchange. That the two terms are renormalized here in a similar way may be a feature of the Holstein-Hubbard model, where the phonon term is coupled to the occupation of a local charge. A model in which the phonons are coupled to a redistribution of the local charge, as with a coupling to Jahn-Teller modes, might behave differently. This topic deserves further investigation.

In calculating the individual contributions to the total energy in the different ordered states and the normal state, we have been able to show the subtle interplay of the various terms. These vary in the weak and strong coupling regimes, and may change discontinuously at the transition. They also depend on the phonon frequency $\omega_{0}$. In the weak coupling regime the energy gain in the broken symmetry state is via a reduction of the potential energy relative to that of the normal state, whereas at strong coupling it is the kinetic energy which is lower in the ordered state. This appears to be a general feature.

The final part of this study has been concerned with the spectra, both of the electrons and phonons. The main effects seen in the phonon spectra are a softening and a broadening of the phonon mode in the region of the transition. In the AFM and CO states well away from the transition there is little effect of the coupling to the electrons on the phonon spectrum. It is more difficult to summarize the results for the electron spectra, as there significant differences develop on all energy scales as the interaction parameters are varied, and as the long range AFM or CO develops. For relatively small values of $U$ and $\lambda$ the main differences are in the region near the Fermi level for AFM or CO states compared with the normal state. This is due to the development of the sublattice structure. For large values of $U$ and small values of $\lambda$ there is the triple peak structure of the Hubbard model, with the narrow renormalized quasiparticle band at the Fermi level flanked by the broadened 'atomic' peaks. As $\lambda$ is increased to $\lambda \sim U$, in the normal state, the narrow quasiparticle band persists, though broadened somewhat, and the atomic-like peaks broadened into shoulders. In the AFM or CO states, the quasiparticle band at the Fermi level develops the features associated with the sublattice structure, as in the weak coupling case. This is also accompanied by much larger shifts of spectral weight on the high energy scales in the sublattice spectral density.

\section{Acknowledgment}

We wish to thank O. Gunnarsson, R. Zeyher for helpful discussions, W. Koller and D. Meyer for their earlier contributions to the development of the NRG programs, and F. Trousselet for critically reading the manuscript.

\section{Appendix A: Mean field theory in the adiabatic limit}

For the mean field theory in the adiabatic limit, the starting point is the Hamiltonian in the form

$$
\begin{aligned}
H= & -t \sum_{i, j, \sigma}\left(c_{i, \sigma}^{\dagger} c_{j, \sigma}+\text { h.c. }\right)+U \sum_{i} \hat{n}_{i, \uparrow} \hat{n}_{i, \downarrow} \\
& +g_{\mathrm{F}} \sum_{i} \hat{x}_{i}\left(\sum_{\sigma} \hat{n}_{i, \sigma}-1\right)+\sum_{i} \frac{\hat{p}_{i}^{2}}{2 M}+\frac{k}{2} \hat{x}_{i}^{2},
\end{aligned}
$$

where the parameters of (A1) and (1) are related by $\omega_{0}=\sqrt{k / M}, g_{\mathrm{F}}=\sqrt{2 \omega_{0}} g$, and $\lambda=g_{\mathrm{F}}^{2} / k$. In this Hamiltonian we can take the limit $M \rightarrow \infty$, such that the kinetic term for the phonons vanishes and we replace the operator $\hat{x}_{i}$ by a static field $x_{i}$. From this we obtain in mean field theory the potential

$$
V\left(x_{i}\right)=\sum_{i} \frac{k}{2} x_{i}^{2}+g_{\mathrm{F}} \sum_{i} x_{i}\left(n_{i}-1\right)+E_{\mathrm{kin}}+E_{U}
$$

where $n_{i}=\sum_{\sigma}\left\langle\hat{n}_{i, \sigma}\right\rangle$. The condition for a local minimum $\partial V\left(x_{i}\right) / \partial x_{i}=0$ yields,

$$
x_{i}=-\frac{g_{\mathrm{F}}}{k}\left(n_{i}-1\right) .
$$

We restrict ourselves to homogeneous solutions, and from the Hamiltonian the mean field self-energy can be read off,

$$
\Sigma_{\alpha, \sigma}(\omega)=U n_{-\sigma}^{\alpha}-\lambda\left(n^{\alpha}-1\right),
$$

independent of $\omega$ and we have employed (A3) for the second term. The index $\alpha=A, B$ corresponds to the sublattice and $\sigma$ to the spin.

In order to determine $n_{\sigma}^{\alpha} \equiv\left\langle\hat{n}_{\sigma}^{\alpha}\right\rangle$ we need to consider the equation

$n_{\sigma}^{\alpha}=\frac{2}{N} \sum_{\boldsymbol{k}}\left\langle c_{\alpha, \boldsymbol{k}, \sigma}^{\dagger} c_{\alpha, \boldsymbol{k}, \sigma}\right\rangle=\frac{2}{N} \sum_{\boldsymbol{k}} \int_{-\infty}^{\infty} \mathrm{d} \omega f_{-}(\omega) \rho_{\alpha, \boldsymbol{k}, \sigma}(\omega)$,

where

$$
\rho_{\alpha, \boldsymbol{k}, \sigma}(\omega)=-\operatorname{Im} \frac{\zeta_{\bar{\alpha}, \sigma}\left(\omega^{+}\right) / \pi}{\zeta_{A, \sigma}\left(\omega^{+}\right) \zeta_{B, \sigma}\left(\omega^{+}\right)-\varepsilon_{\boldsymbol{k}}^{2}},
$$

with $\omega^{+}=\omega+i \eta$. We have used the matrix Green's function for the bipartite lattice in the form (2). This is most easily evaluated with the identity

$$
-\operatorname{Im} \frac{\zeta_{\bar{\alpha}, \sigma}\left(\omega^{+}\right) / \pi}{\zeta_{A, \sigma}\left(\omega^{+}\right) \zeta_{B, \sigma}\left(\omega^{+}\right)-\varepsilon_{\boldsymbol{k}}^{2}}=\sum_{m= \pm} u_{m, \sigma}^{\alpha}\left(\varepsilon_{\boldsymbol{k}}\right) \delta\left[\omega-\omega_{m, \sigma}\left(\varepsilon_{\boldsymbol{k}}\right)\right]
$$

The excitation $\omega_{m, \sigma}\left(\varepsilon_{\boldsymbol{k}}\right)$ are determined from the poles of the Green's function,

$$
\zeta_{A, \sigma}(\omega) \zeta_{B, \sigma}(\omega)-\varepsilon_{\boldsymbol{k}}^{2}=0,
$$


which yields generally

$$
\omega_{ \pm, \sigma}\left(\varepsilon_{\boldsymbol{k}}\right)=\frac{\Sigma_{A, \sigma}-\mu_{A, \sigma}+\Sigma_{B, \sigma}-\mu_{B, \sigma}}{2} \pm E_{\boldsymbol{k}, \sigma},
$$

where

$$
E_{\boldsymbol{k}, \sigma}=\sqrt{\varepsilon_{\boldsymbol{k}}^{2}+\frac{\left[\mu_{A, \sigma}-\Sigma_{A, \sigma}-\left(\mu_{B, \sigma}-\Sigma_{B, \sigma}\right)\right]^{2}}{4}} .
$$

The weights $u_{m, \sigma}^{\alpha}\left(\varepsilon_{\boldsymbol{k}}\right)$ are generally given by the inverse of the derivative w.r.t $\omega$ of

$$
f_{\sigma}^{\alpha}(\omega)=\zeta_{\alpha, \sigma}(\omega)-\frac{\varepsilon_{\boldsymbol{k}}^{2}}{\zeta_{\bar{\alpha}, \sigma}(\omega)}
$$

evaluated at $\omega_{ \pm, \sigma}\left(\varepsilon_{\boldsymbol{k}}\right)$,

$$
u_{m, \sigma}^{\alpha}\left(\varepsilon_{\boldsymbol{k}}\right)=\frac{\zeta_{\bar{\alpha}, \sigma}\left(\omega_{m, \sigma}\left(\varepsilon_{\boldsymbol{k}}\right)\right)^{2}}{\varepsilon_{\boldsymbol{k}}^{2}+\zeta_{\bar{\alpha}, \sigma}\left(\omega_{m, \sigma}\left(\varepsilon_{\boldsymbol{k}}\right)\right)^{2}} .
$$

Using these result we find

$$
n_{\sigma}^{\alpha}=\sum_{m} \int \mathrm{d} \varepsilon \frac{\rho_{0}(\varepsilon) u_{m, \sigma}^{\alpha}(\varepsilon)}{1+\mathrm{e}^{\beta \omega_{m, \sigma}(\varepsilon)}}
$$

through which $n_{\sigma}^{\alpha}$ can be determined self-consistently.

Once $n_{\sigma}^{\alpha}$ is determined we can calculate the ground state energy to determine which state has the lowest energy. The expression for the total energy reads

$$
\begin{aligned}
E_{\mathrm{mf}}= & \frac{1}{N} \sum_{\boldsymbol{k}, \sigma}\left(\varepsilon_{\boldsymbol{k}}\left\langle c_{A, \boldsymbol{k}, \sigma}^{\dagger} c_{B, \boldsymbol{k}, \sigma}\right\rangle+\text { h.c. }\right) \\
& -\frac{\lambda}{2} \sum_{\alpha}\left(n^{\alpha}-1\right)^{2}+\frac{U}{2} \sum_{\alpha} n_{\alpha, \uparrow} n_{\alpha, \downarrow},
\end{aligned}
$$

where we have substituted (A3) for $x$. This can also be written as

$$
\begin{aligned}
E_{\mathrm{mf}}= & E_{\mathrm{kin}}^{\mathrm{mf}}-\lambda\left[\left(\Phi_{\mathrm{co}}^{A}\right)^{2}+\left(\Phi_{\mathrm{co}}^{B}\right)^{2}\right] \\
& +\frac{U}{2}\left(\frac{n_{A}^{2}}{4}-m_{A}^{2}+\frac{n_{B}^{2}}{4}-m_{B}^{2}\right),
\end{aligned}
$$

where

$E_{\mathrm{kin}}^{\mathrm{mf}}=\sum_{\sigma} \int \mathrm{d} \varepsilon \frac{\rho_{0}(\varepsilon) \varepsilon^{2}}{2 E_{\sigma}(\varepsilon)}\left(\frac{1}{1+\mathrm{e}^{\beta \omega_{+, \sigma}(\varepsilon)}}-\frac{1}{1+\mathrm{e}^{\beta \omega_{-, \sigma}(\varepsilon)}}\right)$.

For half filling, $\Phi_{\mathrm{co}}=\left|\Phi_{\mathrm{co}}^{A}\right|=\left|\Phi_{\mathrm{co}}^{B}\right|, \Phi_{\mathrm{afm}}=\left|\Phi_{\mathrm{afm}}^{A}\right|=$ $\left|\Phi_{\mathrm{afm}}^{B}\right|$ this can be written in the simple form

$$
E_{\mathrm{mf}}=E_{\mathrm{kin}}^{\mathrm{mf}}+(U-2 \lambda) \Phi_{\mathrm{co}}^{2}-U \Phi_{\mathrm{afm}}^{2}+\frac{U}{4}
$$

From this we can see that if the order parameters are equal and exclusive the $\mathrm{CO}$ state has lower energy for $\lambda>U$ and the AFM state otherwise.

\section{Appendix B: Calculation of the self-energy}

In NRG calculations it is common practice to determine the self-energy from the Green's function $G_{\alpha, \sigma}(\omega)$ and the higher order Green's function $F_{\alpha, \sigma}(\omega)$ via $^{52}$

$$
\Sigma_{\alpha, \sigma}(\omega)=U \frac{F_{\alpha, \sigma}(\omega)}{G_{\alpha, \sigma}(\omega)} .
$$

This can be derived in an equations of motion approach. As $F, G$ are complex functions, $F=F^{\mathrm{R}}+i F^{\mathrm{I}}$, we can write

$$
\Sigma=U \frac{F^{\mathrm{R}} G^{\mathrm{R}}+F^{\mathrm{I}} G^{\mathrm{I}}+i\left(F^{\mathrm{I}} G^{\mathrm{R}}-F^{\mathrm{R}} G^{\mathrm{I}}\right)}{\left(G^{\mathrm{R}}\right)^{2}+\left(G^{\mathrm{I}}\right)^{2}}
$$

where we have omitted the indices and the arguments. The procedure (B1) for obtaining $\Sigma$ has turned out to work well in many cases both for impurity models and lattice models within the DMFT framework $\underline{38}$ The imaginary part of the retarded self-energy has the well-known property $\operatorname{Im} \Sigma_{\alpha, \sigma}(\omega)<0$, which is respected in Eq. (B2) if $F$ and $G$ are the exact Green's functions. However, in a numerical self-consistent DMFT calculation of $G, F$ small inaccuracies - usually near $|\omega|=0$ - can lead to $F^{\mathrm{I}}(\omega) G^{\mathrm{R}}(\omega)-F^{\mathrm{R}}(\omega) G^{\mathrm{I}}(\omega)>0$ and thus slightly positive values for $\operatorname{Im} \Sigma$ via Eq. (B2). Clearly this is physically incorrect. We have used two different ad-hoc procedures to deal with this complication. The first one (a) is to subtract from $\Sigma_{\alpha, \sigma}^{\mathrm{I}}(\omega)$ the values by which it exceeds zero in a certain interval around $\omega=0$. The second one (b) is to cut-off $\Sigma_{\alpha, \sigma}^{\mathrm{I}}(\omega)$ at zero, i.e. to set it equal to zero for all values where it is positive. We found that in most cases the procedures give approximately the same result. However, very close to the AFM-CO transition the procedure can have an effect on the final result obtained via the self-consistency equation. One finds that ordered solutions are a bit less stable for method (a). We have decided to present all results in this paper obtained by using method (b).
1 M. Imada, A. Fujimori, and Y. Tokura, Rev. Mod. Phys. 70, 1039 (1998).
2 A. Damascelli, Z. Hussain, and Z.-X. Shen, Rev. Mod. Phys. 75, 473 (2003). 
${ }^{3}$ O. Gunnarson, Rev. Mod. Phys. 69, 575 (1997).

4 A. J. Millis, P. B. Littlewood, and B. I. Shraiman, Phys. Rev. Lett. 74, 5144 (1995).

5 A. J. Millis, Nature 392, 147 (1998).

6 B. Powell and R. H. McKenzie, J. Phys.: Cond. Mat. 18, 827 (2006).

7 J. K. Freericks, M. Jarrell, and D. J. Scalapino, Phys. Rev. B 48, 6302 (1993).

8 J. K. Freericks and M. Jarrell, Phys. Rev. B 50, 6939 (1994).

9 J. K. Freericks and M. Jarrell, Phys. Rev. Lett. 75, 2570 (1995).

10 P. Benedetti and R. Zeyher, Phys. Rev. B 58, 14320 (1998).

11 S. Ciuchi and F. de Pasquale, Phys. Rev. B 59, 5431 (1999).

12 J. E. Han and O. Gunnarson, Phys. Rev. B 61, 8628 (2000).

13 A. Deppeler and A. J. Millis, Phys. Rev. B 65, 224301 (2002).

14 A. Deppeler and A. J. Millis, Phys. Rev. B 65, 100301 (2002).

15 M. Capone and S. Ciuchi, Phys. Rev. Lett. 91, 186405 (2003).

16 W. Koller, D. Meyer, Y. Ono, and A. C. Hewson, Europhys. Lett. 66, 559 (2004).

17 W. Koller, D. Meyer, and A. C. Hewson, Phys. Rev. B 70, 155103 (2004).

18 W. Koller, A. C. Hewson, and D. M. Edwards, Phys. Rev. Lett. 95, 256401 (2005).

19 G. Sangiovanni, M. Capone, C. Castellani, and M. Grilli, Phys. Rev. Lett. 94, 026401 (2005).

20 W. von der Linden, E. Berger, and P. Valasek, J. Low Temp. Phys. 99, 517 (1995).

${ }^{21}$ Y. Takada, J. Phys. Soc. Japan 65, 1544 (1996).

22 D. Meyer, A. Hewson, and R. Bulla, Phys. Rev. Lett. 89, 196401 (2002).

23 J. Hague and N. d'Abrumenil, J. Low Temp. Phys. 151, 1149 (2008).

24 G. S. Jeon, T.-H. Park, J. H. Han, H. C. Lee, and H.-Y. Choi, Phys. Rev. B 70, 125114 (2004).

25 T. Holstein, Ann. Phys. (N.Y.) 8, 325 (1959).

${ }^{26}$ G. Sangiovanni, O. Gunnarsson, E. Koch, C. Castellani, and M. Capone, Phys. Rev. Lett. 97, 046404 (2006).

27 R. P. Hardikar and R. T. Clay, Phys. Rev. B 75, 245103 (2007).

${ }^{28}$ G. H. H. Fehske and E. Jeckelmann, Europhys. Lett. 84, 57001 (2008).

29 R. T. Clay and R. P. Hardikar, Phys. Rev. Lett. 95, 096401
(2005).

30 H. Fehske and E. Jeckelmann, in Polarons in Bulk Materials and Systems with Reduced Dimensionality (Proc. Int. School of Physics "Enrico Fermi"), edited by G. Iadonisi, J. Ranninger, and G. de Filippis (IOS Press, Amsterdam, 2006), p. 297.

31 K.-M. Tam, S.-W. Tsai, D. K. Campbell, and A. H. Castro Neto, Phys. Rev. B 75, 161103 (2007).

32 W. Metzner and D. Vollhardt, Phys. Rev. Lett. 62, 324 (1989).

33 A. Georges, G. Kotliar, W. Krauth, and M. Rozenberg, Rev. Mod. Phys. 68, 13 (1996).

34 A. C. Hewson and D. Meyer, J. Phys.: Cond. Mat. 14, 427 (2002).

35 R. Zitzler, T. Pruschke, and R. Bulla, Eur. Phys. J. B 27, 473 (2002).

36 J. Bauer and A. C. Hewson, Eur. Phys. J. B 57, 235 (2007).

37 K. Wilson, Rev. Mod. Phys. 47, 773 (1975).

38 R. Bulla, T. Costi, and T. Pruschke, Rev. Mod. Phys. 80, 395 (2008).

39 R. Peters, T. Pruschke, and F. B. Anders, Phys. Rev. B 74, 245114 (2006).

40 A. Weichselbaum and J. von Delft, Phys. Rev. Lett. 99, 076402 (2007).

41 F. B. Anders and A. Schiller, Phys. Rev. Lett. 95, 196801 (2005).

42 J. Bauer (2009), cond-mat/0907.3751.

43 O. Gunnarson, Alkali-Doped Fullerides: Narrow-Band Solids with Unusual Properties (World Scientific, Singapore, 2004).

44 P. G. J. van Dongen, Phys. Rev. Lett. 67, 757 (1991).

45 R. Micnas, J. Ranninger, and S.Robaszkiewicz, Rev. Mod. Phys. 62, 113 (1990).

46 J. Bauer and A. C. Hewson, phys. stat. sol. (b) 247, 638 (2010).

47 S. Kumar and J. van den Brink, Phys. Rev. B 78, 155123 (2008).

48 J. Bauer, A. C. Hewson, and N. Dupuis, Phys. Rev. B 79, 214518 (2009).

49 A. C. Hewson, A. Oguri, and D. Meyer, Eur. Phys. J. B 40, 177 (2004).

50 W. Hofstetter, Phys. Rev. Lett. 85, 1508 (2000).

51 A. C. Hewson and J. Bauer, J. Phys.: Cond. Mat. 22, 115602 (2010).

52 R. Bulla, A. C. Hewson, and T. Pruschke, J. Phys.: Cond. Mat. 10, 8365 (1998). 